\title{
Critical age-dependent branching Markov processes and their scaling limits
}

\author{
KRISHNA B ATHREYA ${ }^{1,3}$, SIVA R ATHREYA ${ }^{2}$ and \\ SRIKANTH K IYER ${ }^{3}$ \\ ${ }^{1}$ Department of Mathematics and Statistics, Iowa State University, Ames, Iowa 50011, \\ USA \\ ${ }^{2}$ Indian Statistical Institute, 8th Mile, Mysore Road, Bangalore 560 059, India \\ ${ }^{3}$ Department of Mathematics, Indian Institute of Science, Bangalore 560 012, India \\ E-mail: kba@iastate.edu; athreya@isibang.ac.in; skiyer@math.iisc.ernet.in
}

MS received 12 June 2009; revised 15 March 2010

\begin{abstract}
This paper studies: (i) the long-time behaviour of the empirical distribution of age and normalized position of an age-dependent critical branching Markov process conditioned on non-extinction; and (ii) the super-process limit of a sequence of agedependent critical branching Brownian motions.
\end{abstract}

Keywords. Age-dependent; branching; ancestoral times; measure-valued; empirical distribution.

\section{Introduction}

An age-dependent branching Markov process consists of a finite collection of particles distributed in $\mathbb{R}$ in which each particle lives for a random length of time and upon its death gives rise to a random number of offspring. Further, during its lifetime the offspring migrates according to a prescribed Markov process starting from the position where its parent died. The motion process, offspring distribution and lifetime distribution are all independent of each other.

In our model the motion process is allowed to depend on the age of the particle. The lifetimes are not necessarily exponential. The state space $\mathbb{R}$ is chosen mainly for technical convenience and plays no role in the proofs of our results. We assume that the system is critical, i.e. the mean of the offspring distribution is one. We shall describe the model more precisely in the next section.

We study three aspects of such a system. First, at time $t$, conditioned on non-extinction (as such systems die out w.p. 1) we consider a randomly chosen individual from the population. We show that asymptotically (as $t \rightarrow \infty$ ), the joint distribution of the position (appropriately scaled) and age (unscaled) of a randomly chosen individual decouples (see Theorem 1.1). Second, it is shown that conditioned on non-extinction at time $t$, the empirical joint distribution of the age and the normalized position of the population converges in law as $t \rightarrow \infty$ to a random measure characterized by its moments (see Theorem 1.2). Finally, we establish a super-process type limit in a special case. We assume that the motion is governed by an age dependent diffusion and the lifetimes are exponential (see Theorem 1.3). 
Biological applications have motivated the study of branching Markov processes. Branching Brownian motion where the branching part evolves as a standard Markov branching process, lifetimes are exponential and the movement of each individual is Brownian motion has been studied in [13]. More recently, Athreya and Kang [2] studied the case of discrete time Galton-Watson branching process where the movement was modelled as a positive recurrent Markov chain. The above papers focussed on the supercritical case, where the population size diverges to infinity over time. There are many situations, such as in population genetics (see $[12,15])$ where the population evolution is not supercritical but critical, wherein the population dies out in finite time with probability one. However, conditioned on non-extinction, the appropriately normalized process has a limiting distribution, called the Yaglom-limit (see [5] or [17]). The study of the size, age and location spread of such population is of interest.

Limit theorems for critical branching Markov processes where the motion depends on the age does not seem to have been considered in the literature before. These are addressed in this paper.

\subsection{The model}

We begin with the description of the particle system. Suppose we are given the following:

(i) Lifetime distribution $G(\cdot)$. Let $G(\cdot)$ be a cumulative distribution function on $[0, \infty)$, with $G(0)=0$. Let $\mu=\int_{0}^{\infty} s \mathrm{~d} G(s)$, and assume $\int_{0}^{\infty} s^{2} \mathrm{~d} G(s)<\infty$.

(ii) Offspring distribution $\mathbf{p}$. Let $\mathbf{p} \equiv\left\{p_{k}\right\}_{k \geq 0}$ be a probability distribution such that $p_{1}<1, m=\sum_{k=0}^{\infty} k p_{k}=1$ and that $\sigma^{2}=\sum_{k=0}^{\infty} k^{2} p_{k}-1<\infty$.

(iii) Motion Process $\eta(\cdot)$. Let $\eta(\cdot)$ be a $\mathbb{R}$ valued time-inhomogeneous Markov process starting at 0 . We assume that for all $0 \leq t<\infty$,

$$
\begin{aligned}
& E(\eta(t))=0, v(t) \equiv E\left(\eta^{2}(t)\right)<\infty, \sup _{0 \leq s \leq t} v(s)<\infty, \\
& \text { and } \psi=\int_{0}^{\infty} v(s) G(\mathrm{~d} s)<\infty .
\end{aligned}
$$

\section{Branching Markov process $(G, \mathbf{p}, \eta)$}

Suppose we start with an initial configuration $\mathcal{C}_{0}=\left\{\left(a_{0}^{i}, X_{0}^{i}\right): i=1,2, \ldots, N_{0}\right\}, N_{0}<\infty$. $a_{0}^{i}, X_{0}^{i}$ respectively denote the age and position of the $i$-th particle at time 0 . The $i$-th particle present in the system at time 0 lives for a random length of time $L_{i}$ with distribution $G$ and upon its death gives rise to a random number of offspring $\xi$ with distribution $\mathbf{p}$. During its lifetime $L$ the particle moves in $\mathbb{R}$, according to the process $\{x+\eta(t): 0 \leq t \leq L\}$, where $x$ denotes the position of its parent at the time of its birth. More precisely, if an individual is born at time $\tau$ and at location $x$ and has lifetime $L$, then it moves during $[\tau, \tau+L)$ and its movement $\{X(t): \tau \leq t<\tau+L\}$ is distributed as $\{x+\eta(t-\tau): \tau \leq t<\tau+L\}$ and thus the movement of any individual is a random function of its age. We assume that the three objects $(L, \xi, \eta)$ associated with each particle are independent and the family of triplets $(L, \xi, \eta)$ over all particles in the system are i.i.d.

Let $N_{t}$ be the number of particles alive at time $t$ and

$$
\mathcal{C}_{t}=\left\{\left(a_{t}^{i}, X_{t}^{i}\right): i=1,2, \ldots, N_{t}\right\}
$$


denote the age and position configuration of all the individuals alive at time $t$. Since $m=1$ and $G(0)=0$, there is no explosion in finite time (i.e. $P\left(N_{t}<\infty\right)=1$ ). Also $P(\eta(L) \in \mathbb{R})=1$ for each particle. Thus $\mathcal{C}_{t}$ is well-defined for each $0 \leq t<\infty$.

Notation 1.1. For a particle chosen at random from those alive at time $t$, let $M_{t}$, $\left\{L_{t i},\left\{\eta_{t i}(u), 0 \leq u \leq L_{t i}\right\}: 1 \leq i \leq M_{t}\right\}$, denote the number of ancestors, the lifetimes, and the motion processes of its ancestors respectively and $\left\{\eta_{t\left(M_{t}+1\right)}(u): 0 \leq u \leq\right.$ $\left.t-\sum_{i=1}^{M_{t}} L_{t i}\right\}$ be the motion of the individual concerned. If $M_{t}=0$, then

$$
a_{t}=a+t \quad \text { and } \quad X_{t}=\eta_{t 1}(a+t)-\eta_{t 1}(a)+X_{0},
$$

where $a$ and $X_{0}$ are the age and the position of the particle at time $t=0$. If $M_{t}>0$, then the age and position $\left(a_{t}, X_{t}\right)$, of the particle are given by

$$
a_{t}=t-\sum_{i=1}^{M_{t}} L_{t i}+a
$$

and

$$
X_{t}=X_{0}-\eta_{t 1}(a)+\sum_{i=1}^{M_{t}} \eta_{t i}\left(L_{t i}\right)+\eta_{t\left(M_{t}+1\right)}\left(a_{t}\right) .
$$

Note that given $\left\{M_{t}, L_{t i}, 1 \leq i \leq M_{t}\right\}$, the collection of stochastic processes $\left\{\eta_{t i}(u)\right.$, $\left.0 \leq u \leq L_{t i}, 1 \leq i \leq M_{t}\right\}$ have the same distribution as $\{\eta(u): 0 \leq u \leq L\}$ and are independent of each other.

The above model of the particle movement may be contrasted with the models considered in the superprocess literature (see [7]). In those models, given the full branching tree generated by the offspring distribution $\mathbf{p}$ and the lifetime distribution $G$, the motion process for each line of descent is a Markov process $\tilde{\eta}$ with a given transition function. Thus, the position of a sampled individual at time $t$ has the same distribution as $\tilde{\eta}(t)$ and this is different from (1.5).

Let $\mathcal{B}\left(\mathbb{R}_{+}\right)$(and $\mathcal{B}(\mathbb{R})$ ) be the Borel $\sigma$-algebra on $\mathbb{R}_{+}$(and $\mathbb{R}$ ). Let $M\left(\mathbb{R}_{+} \times \mathbb{R}\right.$ ) be the space of finite Borel measures on $\mathbb{R}_{+} \times \mathbb{R}$ equipped with the weak topology. Let $M_{a}\left(\mathbb{R}_{+} \times \mathbb{R}\right):=\left\{v \in M\left(\mathbb{R}_{+} \times \mathbb{R}\right): v=\sum_{i=1}^{n} \delta_{a_{i}, x_{i}}(\cdot, \cdot), n \in \mathbb{N}, a_{i} \in \mathbb{R}_{+}, x_{i} \in \mathbb{R}\right\}$. For any set $A \in \mathcal{B}\left(\mathbb{R}_{+}\right)$and $B \in \mathcal{B}(\mathbb{R})$, let $Y_{t}(A \times B)$ be the number of particles at time $t$ whose ages are in $A$ and positions in $B$. As pointed out earlier, $m<\infty, G(0)=0$ implies that $Y_{t} \in M_{a}\left(\mathbb{R}_{+} \times \mathbb{R}\right)$ for all $t>0$ if $Y_{0}$ does so. Fix a function $\phi \in C_{b}^{+}\left(\mathbb{R}_{+} \times \mathbb{R}\right)$ (the set of all bounded, continuous and positive functions from $\mathbb{R}_{+} \times \mathbb{R}$ to $\mathbb{R}_{+}$), and define

$$
\left\langle\phi, Y_{t}\right\rangle \equiv \int \phi \mathrm{d} Y_{t}=\sum_{i=1}^{N_{t}} \phi\left(a_{t}^{i}, X_{t}^{i}\right) .
$$

Since $\eta(\cdot)$ is a Markov process, it can be seen that $\left\{Y_{t}: t \geq 0\right\}$ is a Markov process and we shall call $Y \equiv\left\{Y_{t}: t \geq 0\right\}$ the $(G, \mathbf{p}, \eta)$ - branching Markov process.

Note that $\mathcal{C}_{t}$ determines $Y_{t}$ and conversely. The Laplace functional of $Y$, is given by

$$
\begin{aligned}
L_{t} \phi(a, x) & :=E_{a, x}\left[\mathrm{e}^{-\left\langle\phi, Y_{t}\right\rangle}\right] \\
& \equiv E\left[\mathrm{e}^{-\left\langle\phi, Y_{t}\right\rangle} \mid Y_{0}=\delta_{a, x}\right], \quad t \geq 0, \quad \text { and } \phi \in C_{b}^{+}\left(\mathbb{R}_{+} \times \mathbb{R}\right) .
\end{aligned}
$$


From the independence intrinsic in $\left\{Y_{t}: t \geq 0\right\}$, we have

$$
E_{v_{1}+v_{2}}\left[\mathrm{e}^{-\left\langle\phi, Y_{t}\right\rangle}\right]=\left(E_{v_{1}}\left[\mathrm{e}^{-\left\langle\phi, Y_{t}\right\rangle}\right]\right)\left(E_{\nu_{2}}\left[\mathrm{e}^{-\left\langle\phi, Y_{t}\right\rangle}\right]\right),
$$

where $E_{v}\left[\mathrm{e}^{-\left\langle\phi, Y_{t}\right\rangle}\right]:=E\left[\mathrm{e}^{-\left\langle\phi, Y_{t}\right\rangle} \mid Y_{0}=v\right]$ for $v \in M_{a}\left(\mathbb{R}_{+} \times \mathbb{R}\right)$. This is usually referred to as the branching property of $Y$. In particular, if $v=\sum_{i=1}^{n} \delta_{a_{i}, x_{i}}, n<\infty$, then (see [10])

$$
E_{\nu}\left[\mathrm{e}^{-\left\langle\phi, Y_{t}\right\rangle}\right]=\prod_{i=1}^{n} L_{t} \phi\left(a_{i}, x_{i}\right)=\mathrm{e}^{\left\langle\log L_{t} \phi, v\right\rangle} .
$$

\subsection{Main results}

In this section we describe the main results of the paper. Let $A_{t}$ be the event $\left\{N_{t}>0\right\}$, where $N_{t}$ is the number of particles alive at time $t$. As $p_{0}<1, P\left(A_{t}\right)>0$ for all $0 \leq t<\infty$ provided $P\left(N_{0}=0\right) \neq 1$. Let $\psi$ be as defined in (1.1).

Theorem 1.1 (Limiting behaviour of a randomly chosen particle). On the event $A_{t}=$ $\left\{N_{t}>0\right\}$, let $\left(a_{t}, X_{t}\right)$ be the age and position of a particle chosen uniformly from those alive at time $t$. Assume (1.1). Then, conditioned on $A_{t},\left(a_{t}, \frac{X_{t}}{\sqrt{t}}\right)$ converges as $t \rightarrow \infty$, to $(U, V)$ in distribution, where $U$ and $V$ are independent with $U$ a strictly positive, absolutely continuous random variable with density given by $\frac{1}{\mu}(1-G(\cdot))$ and $V$ is normally distributed with mean 0 and variance $\frac{\psi}{\mu}$.

Next consider the scaled empirical measure $\tilde{Y}_{t} \in M_{a}\left(\mathbb{R}_{+} \times \mathbb{R}\right)$ given by $\tilde{Y}_{t}(A \times B) \equiv$ $Y_{t}(A \times \sqrt{t} B), A \in \mathcal{B}\left(\mathbb{R}_{+}\right), B \in \mathcal{B}(\mathbb{R})$.

Theorem 1.2 (Empirical measure). Assume (1.1). Then, conditioned on $A_{t}=\left\{N_{t}>0\right\}$, the random measures $\left\{\frac{\tilde{Y}_{t}}{N_{t}}\right\}$ converge as $t \rightarrow \infty$ in distribution to a random measure $v$, characterized by its moment sequence $m_{k}(\phi) \equiv E\left[\langle v, \phi\rangle^{k}\right]$, for $\phi \in C_{b}^{+}\left(\mathbb{R}_{+} \times \mathbb{R}\right), k \geq 1$.

An explicit formula for $m_{k}(\phi)$ is given in (4.3) below.

\section{Remark 1.1.}

(i) Theorems 1.1 and 1.2 can also be extended to the case when $\eta\left(L_{1}\right)$, with $L_{1} \stackrel{d}{=} G$, is in the domain of attraction of a stable law of index $0<\alpha \leq 2$.

(ii) Equations (1.4) and (1.5) suggest that Theorem 1.1 should follow from the central limit theorem. But conditioning on non-extinction introduces dependencies in the lifetimes of the ancestors of the chosen individual. To take care of this we prove in $\S 2$ four propositions on age-dependent branching processes which are used in proving the result (see $\S 3$ ).

We now study the super-process scaling limit for the age-dependent branching process. For a review of the super-process literature we refer the reader to [7], [10], [11] and [16]. One may view a super-process as a renormalized limit of the empirical measure of a sequence of branching Markov processes. When motion depends on age or in the branching Markov process the position of the parent at the time of its death and the positions of its offspring (at the time of their birth) are not the same, the system is said to be nonlocal. In such systems the standard super-process scaling limit procedure does not work. 
References [6] and [8] have considered two special cases where such a scaling limit has been obtained. In [6] an age-dependent branching process is rescaled (i.e. the particles do not perform any motion) and in [8] a general non-local super-process limit is obtained when the offspring distribution is given by $p_{1}=1$.

In order to obtain a super-process type limit, we scale the age and the motion differently. In the limit we obtain an age- 'averaged' super-process. To illustrate this idea we consider a specific sequence of branching Markov processes $\left(G_{n}, \mathbf{p}_{n}, \eta_{n}\right)_{\{n \geq 1\}}$ denoted by $\left\{Y_{t}^{n}\right.$ : $t \geq 0\}_{\{n \geq 1\}}$ given by:

\section{Notation 1.2.}

(a) Initial measure. For $n \geq 1, Y_{0}^{n}=\pi_{n v}$, where $\pi_{n v}$ is a Poisson random measure with intensity $n v$, for some finite product measure $v=\alpha \times \mu \in M\left(\mathbb{R}_{+} \times \mathbb{R}\right)$.

(b) Lifetime $G^{n}(\cdot)$. For all $n \geq 1, G^{n} \equiv G$ is an exponential distribution with mean $\frac{1}{\lambda}$, $0<\lambda<\infty$.

(c) Offspring distribution $\mathbf{p}_{n}$. For each $n \geq 1$, let $F_{n}(u)=\sum_{k=0}^{\infty} p_{n, k} u^{k}$ be the generating function of the offspring distribution $\mathbf{p}_{n} \equiv\left\{p_{n, k}\right\}_{k \geq 0}$. We shall assume that $F_{n}$ satisfies,

$$
\lim _{n \rightarrow \infty} \sup _{0 \leq u \leq N}\left|n^{2}\left(F_{n}(1-u / n)-(1-u / n)\right)-c u^{2}\right| \rightarrow 0,
$$

for some $c>0$ and all $N>0$.

(d) Motion process $\eta_{n}(\cdot)$. For all $n \geq 1$,

$$
\left\{\eta_{n}(t) \equiv \frac{1}{\sqrt{n}} \int_{0}^{t} \sigma(u) \mathrm{d} B_{n}(u), \quad t \geq 0,\right\}
$$

where $\left\{B_{n}(t): t \geq 0\right\}$ are independent standard Brownian motions starting at 0 and $\sigma: \mathbb{R}_{+} \rightarrow \mathbb{R}$ is a continuous function such that for each $n \geq 1, \eta_{n}$ satisfies (1.1).

We now define the limiting age-averaged super-process.

\section{DEFINITION 1.1}

Let $f \in C_{l}^{+}\left(\mathbb{R}_{+} \times \mathbb{R}\right)$ (the set of all nonnegative, continuous functions from $\mathbb{R}_{+} \times \mathbb{R}$ to $\mathbb{R}$ with finite limits at infinity). Let $\left\{B_{t}: t \geq 0\right\}$ be a standard Brownian motion and $\mathcal{E}$ an exponential random variable with mean $\frac{1}{\lambda}, 0<\lambda<\infty$, independent of $\left\{B_{t}: t \geq 0\right\}$ and let $\psi$ be as defined in (1.1). Let

$$
U_{t} f(x) \equiv E\left(f\left(\mathcal{E}, x+\sqrt{\lambda \psi} B_{t}\right)\right), \quad x \in \mathbb{R}, t \geq 0 .
$$

Let $u_{t}(f)$ be the unique solution of the integral equation

$$
u_{t} f(x)=U_{t} f(x)-c \lambda \int_{0}^{t} U_{t-s}\left(u_{s}(f)^{2}\right)(x) \mathrm{d} s, \quad x \in \mathbb{R}, t \geq 0 .
$$

Let $\mathcal{Y} \equiv\left\{\mathcal{Y}_{t}: t \geq 0\right\}$ be a $M\left(\mathbb{R}_{+} \times \mathbb{R}\right)$-valued Markov process such that $\mathcal{Y}_{0} \equiv \mathcal{E} \times \mu$, i.e. $\mathcal{Y}_{0} \in M\left(\mathbb{R}_{+} \times \mathbb{R}\right)$ and for any $f \in C_{b}\left(\mathbb{R}_{+} \times \mathbb{R}\right)$,

$$
\left\langle f, \mathcal{Y}_{0}\right\rangle=\lambda \int_{\mathbb{R}_{+} \times \mathbb{R}} f(a, x) \mathrm{e}^{-\lambda a} \mathrm{~d} a \mu(\mathrm{d} x),
$$


and whose Laplace functional is given by

$$
E_{\mathcal{E} \times \mu}\left[\mathrm{e}^{-\left\langle f, \mathcal{Y}_{t}\right\rangle}\right] \equiv E\left[\mathrm{e}^{-\left\langle f, \mathcal{Y}_{t}\right\rangle} \mid \mathcal{Y}_{0}=\mathcal{E} \times \mu\right]=\mathrm{e}^{-\left\langle u_{t}(f), \mu\right\rangle} .
$$

The function $u_{s}(f)$ in the second term of (1.11) is interpreted in the natural way as a function on $\mathbb{R}_{+} \times \mathbb{R}$ with $u_{s}(f)(a, x)=u_{s}(f)(x)$ for all $a>0, x \in \mathbb{R}$. It can be shown that there is a unique solution of the nonlinear integral equation (1.11) and there exists a $M\left(\mathbb{R}_{+} \times \mathbb{R}\right)$-valued Markov process $\mathcal{Y}$ satisfying (1.12) (see [7]).

Note that in the process $\mathcal{Y} \equiv\left\{\mathcal{Y}_{t}: t \geq 0\right\}$ defined above, the distribution of the age (i.e. the first coordinate) is exponential and does not change with time. The spatial evolution behaves like that of a super-process where the motion of particles is like that of a Brownian motion with variance equal to the averaged (over lifetime) variance of the age-dependent motion.

Theorem 1.3 (Age structured super-process). Let $\epsilon>0$. Let $\left\{Y_{t}^{n}: t \geq 0\right\}_{n \geq 1}$ be the sequence of branching Markov processes defined above (i.e. in (a), (b), (c) and (d)). Then as $n \rightarrow \infty,\left\{\mathcal{Y}_{t}^{n} \equiv \frac{1}{n} Y_{n t}^{n}, t \geq \epsilon\right\}$ converges weakly on the Skorokhod space $D\left([\epsilon, \infty), M\left(\mathbb{R}_{+} \times \mathbb{R}\right)\right)$ to $\left\{\mathcal{Y}_{t}: t \geq \epsilon\right\}$.

The rest of the paper is organized as follows. In $\S 2$, we prove some preliminary results concerning age-dependent branching processes. Using a central limit type theorem (Proposition 2.3) proved in this section we prove Theorem 1.1 in $\$ 3$. In $\S 2$, we also show that the joint distribution of coalescent times for a sample of $k \geq 1$ individuals chosen at random from the population at time $t$ scaled by $t$ converges, as $t \rightarrow \infty$ (see Theorem 2.1). This result is of independent interest and is a key tool in the proof of Theorem 1.2 in $\S 4$. Finally in $\$ 5$ we prove Theorem 1.3.

\section{Results on branching processes}

Let $\left\{N_{t}: t \geq 0\right\}$ be the age-dependent branching process with offspring distribution $\left\{p_{k}\right\}_{k \geq 0}$ and lifetime distribution $G$ as defined earlier. Let $\left\{\zeta_{k}\right\}_{k \geq 0}$ be the embedded discrete time Galton-Watson branching process with $\zeta_{k}$ the size of the $k$-th generation, $k \geq 0$. Recall that $A_{t}=\left\{N_{t}>0\right\}$. On this event, choose an individual at random with uniform distribution from those alive at time $t$. Let $M_{t}$ be the generation number and $a_{t}$ be the age of this individual, respectively.

\section{PROPOSITION 2.1}

Let $A_{t}, a_{t}, M_{t}$ and $N_{t}$ be as above with $N_{0}=1$. Let $\mu$ and $\sigma$ be as in $\S 1.1$. Then

(a) $\lim _{t \rightarrow \infty} t P\left(A_{t}\right)=\frac{2 \mu}{\sigma^{2}}$;

(b) for all $x>0, \lim _{t \rightarrow \infty} P\left(\frac{N_{t}}{t}>x \mid A_{t}\right)=\mathrm{e}^{-\frac{2 \mu x}{\sigma^{2}}}$;

(c) for all $\epsilon>0, \lim _{t \rightarrow \infty} P\left(\left|\frac{M_{t}}{t}-\frac{1}{\mu}\right|>\epsilon \mid A_{t}\right)=0$;

(d) for all $x>0, \lim _{t \rightarrow \infty} P\left(a_{t} \leq x \mid A_{t}\right)=\frac{1}{\mu} \int_{0}^{x}(1-G(s)) \mathrm{d} s$.

Proof. For (a) and (b), see chapter 4 in [5]. For (c), see [9] and for (d) the proof from [3] for the super-critical case can be adapted. 
PROPOSITION 2.2 (Law of large numbers)

Let $\epsilon>0$ be given. For the randomly chosen individual at time $t$, let $\left\{L_{t i}: 1 \leq i \leq M_{t}\right\}$, be the lifetimes of its ancestors. Let $h:[0, \infty) \rightarrow \mathbb{R}$ be Borel measurable and $E\left(\left|h\left(L_{1}\right)\right|\right)<\infty$ with $L_{1} \stackrel{d}{=} G$. Then, as $t \rightarrow \infty$,

$$
P\left(\left|\frac{1}{M_{t}} \sum_{i=1}^{M_{t}} h\left(L_{t i}\right)-E\left(h\left(L_{1}\right)\right)\right|>\epsilon \mid A_{t}\right) \rightarrow 0 .
$$

Proof. Let $\epsilon$ and $\epsilon_{1}>0$ be given and let $k_{1}(t)=t\left(\frac{1}{\mu}-\epsilon\right)$ and $k_{2}(t)=t\left(\frac{1}{\mu}+\epsilon\right)$. By Proposition 2.1 there exists $\delta>0, \eta>0$ and $t_{0}>0$ such that for all $t \geq t_{0}$,

$$
\begin{aligned}
& t P\left(N_{t}>0\right)>\delta \quad \text { and } P\left(N_{t} \leq t \eta \mid A_{t}\right)<\epsilon_{1} ; \\
& P\left(M_{t} \in\left[k_{1}(t), k_{2}(t)\right]^{c} \mid A_{t}\right)<\epsilon_{1} .
\end{aligned}
$$

Also by the strong law of large numbers for any $\left\{L_{i}\right\}_{i \geq 1}$ i.i.d. $G$ with $E\left|h\left(L_{1}\right)\right|<\infty$,

$$
\lim _{k \rightarrow \infty} P\left(\sup _{j \geq k}\left|\frac{1}{j} \sum_{i=1}^{j} h\left(L_{i}\right)-E\left(h\left(L_{1}\right)\right)\right|>\epsilon\right)=0 .
$$

Let $\left\{\zeta_{k}\right\}_{k \geq 0}$ be the embedded Galton-Watson process. For each $t>0$ and $k \geq 1$, let $\zeta_{k t}$ denote the number of lines of descent in the $k$-th generation alive at time $t$ (i.e. the successive lifetimes $\left\{L_{i}\right\}_{i \geq 1}$ of the individuals in that line of descent satisfying $\sum_{i=1}^{k} L_{i} \leq$ $\left.t \leq \sum_{i=1}^{k+1} L_{i}\right)$. Denote the lines of descent of these individuals by $\left\{\zeta_{k t j}: 1 \leq j \leq \zeta_{k t}\right\}$. Call $\zeta_{k t j}$ bad if

$$
\left.\mid \frac{1}{k} \sum_{i=1}^{k} h\left(L_{k t j i}\right)-E\left(h\left(L_{1}\right)\right)\right) \mid>\epsilon,
$$

where $\left\{L_{k t j i}\right\}_{i \geq 1}$ are the successive lifetimes in the line of descent $\zeta_{k t j}$ starting from the ancestor. Let $\zeta_{k t, b}$ denote the cardinality of the set $\left\{\zeta_{k t j}: 1 \leq j \leq \zeta_{k t}\right.$ and $\zeta_{k t j}$ is bad $\}$. So,

$P$ (the chosen line of descent at time $t$ is $\left.\left.b a d, M_{t} \in\left[k_{1}(t), k_{2}(t)\right]\right) \mid A_{t}\right)$

$$
=\frac{1}{P\left(A_{t}\right)} E\left(\frac{\sum_{j=k_{1}(t)}^{k_{2}(t)} \zeta_{j t, b}}{N_{t}} ; A_{t}\right) \text {. }
$$

Consequently,

$$
\begin{aligned}
P( & \left.\left|\frac{1}{M_{t}} \sum_{i=1}^{M_{t}} h\left(L_{t i}\right)-E\left(h\left(L_{1}\right)\right)\right|>\epsilon \mid A_{t}\right) \\
= & \left.P \text { (the chosen line of descent at time } t \text { is } \mathrm{bad} \mid A_{t}\right) \\
\leq & \left.\left.P \text { (the chosen line of descent at time } t \text { is bad, } M_{t} \in\left[k_{1}(t), k_{2}(t)\right]\right) \mid A_{t}\right) \\
& \left.+P\left(M_{t} \in\left[k_{1}(t)\right), k_{2}(t)\right]^{c} \mid A_{t}\right)
\end{aligned}
$$




$$
\begin{aligned}
= & \left.\frac{1}{P\left(N_{t}>0\right)} E\left(\frac{\sum_{j=k_{1}(t)}^{k_{2}(t)} \zeta_{j t, b}}{N_{t}} ; A_{t}\right)+P\left(M_{t} \in\left[k_{1}(t)\right), k_{2}(t)\right]^{c} \mid A_{t}\right) \\
= & \frac{1}{P\left(N_{t}>0\right)} E\left(\frac{\sum_{j=k_{1}(t)}^{k_{2}(t)} \zeta j t, b}{N_{t}} ; N_{t}>t \eta\right) \\
& +\frac{1}{P\left(N_{t}>0\right)} E\left(\frac{\sum_{j=k_{1}(t)}^{k_{2}(t)} \zeta_{j t, b}}{N_{t}} ; 0<N_{t} \leq t \eta\right) \\
& \left.+P\left(M_{t} \in\left[k_{1}(t)\right), k_{2}(t)\right]^{c} \mid A_{t}\right) \\
\leq & \frac{1}{P\left(N_{t}>0\right)} E\left(\frac{\sum_{j=k_{1}(t)}^{k_{2}(t)} \zeta_{j t, b}}{t \eta} ; N_{t}>t \eta\right)+ \\
& \left.+\frac{P\left(0<N_{t} \leq t \eta\right)}{P\left(N_{t}>0\right)}+P\left(M_{t} \in\left[k_{1}(t)\right), k_{2}(t)\right]^{c} \mid A_{t}\right) \\
= & \frac{1}{t \eta P\left(N_{t}>0\right)} \sum_{j=k_{1}(t)}^{k_{2}(t)} E\left(\zeta_{j t, b}\right) \\
& \left.+P\left(0<N_{t} \leq t \eta \mid N_{t}>0\right)+P\left(M_{t} \in\left[k_{1}(t)\right), k_{2}(t)\right]^{c} \mid A_{t}\right)
\end{aligned}
$$

For $t \geq t_{0}$ by (2.1) and (2.2), the last two terms in (2.5) are less than $\epsilon_{1}$. The first term is equal to

$$
\begin{aligned}
& \frac{1}{t \eta P\left(N_{t}>0\right)} \sum_{j=k_{1}(t)}^{k_{2}(t)} E\left(\zeta_{j t, b}\right) \\
& =\frac{1}{t \eta P\left(N_{t}>0\right)} \sum_{j=k_{1}(t)}^{k_{2}(t)} E\left(\sum_{i=1}^{\zeta_{j}} 1_{\left\{\zeta_{j t i} \text { is bad }\right\}}\right) \\
& =\frac{1}{t \eta P\left(N_{t}>0\right)} \sum_{j=k_{1}(t)}^{k_{2}(t)} E\left(\zeta_{j}\right) \\
& \quad \times P\left(\sum_{i=1}^{j} L_{i} \leq t<\sum_{i=1}^{j+1} L_{i},\left|\frac{1}{j} \sum_{i=1}^{j} h\left(L_{i}\right)-E\left(h\left(L_{1}\right)\right)\right|>\epsilon\right),
\end{aligned}
$$

where the $\left\{L_{i}\right\}_{i \geq 1}$ are i.i.d. $G$. Using (2.1) and (since $\left.m=1\right) E\left(\zeta_{j}\right)=E\left(\zeta_{0}\right)$ we can conclude that

$$
\frac{1}{t \eta P\left(N_{t}>0\right)} \sum_{j=k_{1}(t)}^{k_{2}(t)} E\left(\zeta_{j t, b}\right)
$$




$$
\begin{aligned}
& \leq E\left(\zeta_{0}\right) \frac{P\left(\sup _{j \geq k_{1}(t)}\left|\frac{1}{j} \sum_{i=1}^{j} h\left(L_{i}\right)-E\left(h\left(L_{1}\right)\right)\right|>\epsilon\right)}{t \eta P\left(N_{t}>0\right)} \\
& \leq E\left(\zeta_{0}\right) \frac{P\left(\sup _{j \geq k_{1}(t)}\left|\frac{1}{j} \sum_{i=1}^{j} h\left(L_{i}\right)-E\left(h\left(L_{1}\right)\right)\right|>\epsilon\right)}{\eta \delta}
\end{aligned}
$$

which by (2.3) goes to zero as $t \rightarrow \infty$. So we have shown that

$$
\limsup _{t \rightarrow \infty} P\left(\left|\frac{1}{M_{t}} \sum_{i=1}^{M_{t}} h\left(L_{t i}\right)-E\left(h\left(L_{1}\right)\right)\right|>\epsilon \mid A_{t}\right)<2 \epsilon_{1} .
$$

Since $\epsilon_{1}>0$ is arbitrary, the proof is complete.

In the following, for any collection $\mathcal{A}$ of random variables, $\sigma(\mathcal{A})$ will denote the $\sigma$-algebra generated by $\mathcal{A}$.

\section{PROPOSITION 2.3}

Assume (1.1) holds. Let $\left\{L_{i}\right\}_{i \geq 1}$ be i.i.d. $G$ and $\left\{\eta_{i}\right\}_{i \geq 1}$ be i.i.d. copies of $\eta$ and independent of $\left\{L_{i}\right\}_{i \geq 1}$. For $\theta \in \mathbb{R}, t \geq 0$ define $\phi(\theta, t)=E \mathrm{e}^{i \theta \eta(t)}$. Then there exists an event $D$, with $P(D)=1$ and on $D$, for all $\theta \in \mathbb{R}$,

$$
\prod_{j=1}^{n} \phi\left(\frac{\theta}{\sqrt{n}}, L_{j}\right) \rightarrow \mathrm{e}^{\frac{-\theta^{2} \psi}{2}}, \quad \text { as } n \rightarrow \infty,
$$

where $\psi$ is as in (1.1).

Proof. Recall from (1.1) that $v(t)=E\left(\eta^{2}(t)\right)<\infty$ for $t \geq 0$. By the strong law of large numbers and (1.1),

$$
\frac{\sum_{j=1}^{n} v\left(L_{j}\right)}{n} \rightarrow \psi \quad \text { w.p. } 1 .
$$

Note that,

$$
\prod_{i=1}^{n} \phi\left(\frac{\theta}{\sqrt{\sum_{j=1}^{n} v\left(L_{j}\right)}}, L_{j}\right)=E\left(\mathrm{e}^{i \theta \sum_{j=1}^{n} X_{n j}} \mid \mathcal{F}\right),
$$

where

$$
X_{n i}=\frac{\eta_{i}\left(L_{i}\right)}{\sqrt{\sum_{j=1}^{n} v\left(L_{j}\right)}} \text { for } 1 \leq i \leq n,
$$

and $\mathcal{F} \equiv \sigma\left(L_{i}: i \geq 1\right)$. We need to prove a central limit theorem for the triangular array $\left\{X_{n j}: 1 \leq j \leq n\right\}$. Given $\mathcal{F},\left\{X_{n i}: 1 \leq i \leq n\right\}$ is a triangular array of independent random variables such that for $1 \leq i \leq n, E\left(X_{n i} \mid \mathcal{F}\right)=0, \sum_{i=1}^{n} E\left(X_{n i}^{2} \mid \mathcal{F}\right)=1$.

Let $\epsilon>0$ be given. Let

$$
L_{n}(\epsilon)=\sum_{i=1}^{n} E\left(X_{n i}^{2} ; X_{n i}^{2}>\epsilon \mid \mathcal{F}\right) .
$$


Let $D$ be the event on which (2.7) holds. Using the dominated convergence theorem, we have

$$
\begin{aligned}
\limsup _{n \rightarrow \infty} L_{n}(\epsilon) & \leq \limsup _{n \rightarrow \infty} \frac{2}{\psi n} \sum_{i=1}^{n} E\left(\left|\eta_{i}\left(L_{i}\right)\right|^{2}:\left|\eta_{i}\left(L_{i}\right)\right|^{2}>\frac{\epsilon n \psi}{2} \mid \mathcal{F}\right) \\
& \leq \limsup _{k \rightarrow \infty} \limsup _{n \rightarrow \infty} \frac{2}{\psi n} \sum_{i=1}^{n} E\left(\left|\eta_{i}\left(L_{i}\right)\right|^{2}:\left|\eta_{i}\left(L_{i}\right)\right|^{2}>k \mid \mathcal{F}\right) \\
& \leq \limsup _{k \rightarrow \infty} \frac{2}{\psi} E\left(\left|\eta_{1}\left(L_{1}\right)\right|^{2}:\left|\eta_{1}\left(L_{1}\right)\right|^{2}>k\right) \\
& =0
\end{aligned}
$$

on $D$. Thus, the Lindeberg-Feller central limit theorem (see [4]) implies, that on $D$, for all $\theta \in \mathbb{R}$,

$$
\lim _{n \rightarrow \infty} E\left(\mathrm{e}^{i \theta \sum_{j=1}^{n} X_{n j}} \mid \mathcal{F}\right) \rightarrow \mathrm{e}^{\frac{-\theta^{2}}{2}} .
$$

Combining this with (2.7) and (2.8) we have the result.

\section{PROPOSITION 2.4}

For the randomly chosen individual at time, let $\left\{L_{t i},\left\{\eta_{t i}(u): 0 \leq u \leq L_{t i}\right\}: 1 \leq i \leq M_{t}\right\}$, be the lifetimes and motion processes of its ancestors. Let $H_{t}=\frac{1}{\sqrt{M_{t}}} \sum_{i=1}^{M_{t}} \eta_{t i}\left(L_{t i}\right)$, and $\mathcal{L}_{t} \equiv \sigma\left(M_{t}, L_{t i}: 1 \leq i \leq M_{t}\right)$. Then

$$
E\left(\left|E\left(\mathrm{e}^{i \theta H_{t}} \mid \mathcal{L}_{t}\right)-\mathrm{e}^{-\frac{\theta^{2} \psi}{2}}\right| \mid A_{t}\right) \rightarrow 0 .
$$

Proof. Fix $\theta \in \mathbb{R}, \epsilon_{1}>0$ and $\epsilon>0$. Replace the definition of 'bad' in (2.4) by

$$
\left|\prod_{i=1}^{k} \phi\left(\frac{\theta}{\sqrt{k}}, L_{k t j i}\right)-\mathrm{e}^{-\frac{\theta^{2} \psi}{2}}\right|>\epsilon .
$$

By Proposition 2.3 we have

$$
\lim _{k \rightarrow \infty} P\left(\sup _{j \geq k}\left|\prod_{i=1}^{j} \phi\left(\frac{\theta}{\sqrt{j}}, L_{i}\right)-\mathrm{e}^{-\frac{\theta^{2} \psi}{2}}\right|>\epsilon\right)=0 .
$$

Using this in place of (2.3) and imitating the proof of Proposition 2.2 (since the details mirror that proof we avoid repeating them here), we obtain that for $t$ sufficiently large

$$
P\left(\left|\prod_{i=1}^{M_{t}} \phi\left(\frac{\theta}{\sqrt{M_{t}}}, L_{t i}\right)-\mathrm{e}^{-\frac{\theta^{2} \psi}{2}}\right|>\epsilon_{1} \mid A_{t}\right)<\epsilon .
$$

Now for all $\theta \in \mathbb{R}$,

$$
E\left(\mathrm{e}^{i \theta H_{t}} \mid \mathcal{L}_{t}\right)=\prod_{i=1}^{M_{t}} \phi\left(\frac{\theta}{\sqrt{M_{t}}}, L_{t i}\right) .
$$


So,

$$
\begin{aligned}
& \limsup _{t \rightarrow \infty} E\left(\left|E\left(\mathrm{e}^{i \theta \frac{1}{\sqrt{M_{t}}} \sum_{i=1}^{M_{t}} \eta_{i}\left(L_{t i}\right)} \mid \mathcal{L}_{t}\right)-\mathrm{e}^{-\frac{\theta^{2} \psi}{2}}\right| \mid A_{t}\right) \\
& =\limsup _{t \rightarrow \infty} E\left(\left|\prod_{i=1}^{M_{t}} \phi\left(\frac{\theta}{\sqrt{M_{t}}}, L_{t i}\right)-\mathrm{e}^{-\frac{\theta^{2} \psi}{2}}\right| \mid A_{t}\right) \\
& \quad<\epsilon_{1}+2 \limsup _{t \rightarrow \infty} P\left(\left|\prod_{i=1}^{M_{t}} \phi\left(\frac{\theta}{\sqrt{M_{t}}}, L_{t i}\right)-\mathrm{e}^{-\frac{\theta^{2} \psi}{2}}\right|>\epsilon_{1} \mid A_{t}\right) \\
& =\epsilon_{1}+2 \epsilon .
\end{aligned}
$$

Since $\epsilon>0, \epsilon_{1}>0$ are arbitrary we have the result.

The above four propositions will be used in the proof of Theorem 1.1. For the proof of Theorem 1.2 we will need a result on coalescing times of the lines of descent.

Fix $k \geq 2$. On the event $A_{t}=\left\{N_{t}>0\right\}$, pick $k$ individuals $C_{1}, C_{2}, \ldots, C_{k}$, from those alive at time $t$ by simple random sampling without replacement. For any two particles $C_{i}, C_{j}$, let $\tau_{C_{j}, C_{i}, t}$ be the death time of their most recent common ancestor. Let $\tau_{k-1, t}=$ $\sup \left\{\tau_{C_{j}, C_{i}, t}: i \neq j, 1 \leq i, j \leq k\right\}$. Thus $\tau_{k-1, t}$ is the last time and before $t$ there are $k-1$ ancestors of the $k$ individuals $C_{1}, C_{2}, \ldots, C_{k}$. More generally, for $1 \leq j \leq k-1$, let $\tau_{j, t}$ be the last time before $t$ when there are $j$ ancestors of the $k$ individuals $C_{1}, C_{2}, \ldots, C_{k}$.

\section{Theorem 2.1.}

(i) For any $i, j, \lim _{t \rightarrow \infty} P\left(\frac{{ }^{\tau_{C},}, C_{j}, t}{t} \leq x \mid A_{t}\right) \equiv H(x)$ exists for all $x \geq 0$ and $H(\cdot)$ is an absolutely continuous distribution function on $[0,1]$.

(ii) Conditioned on $A_{t}$ the vector $\tilde{\tau}_{t}=\frac{1}{t}\left(\tau_{j, t}: 1 \leq j \leq k-1\right)$ as $t \rightarrow \infty$ converges in distribution to a random vector $\tilde{T}=\left(T_{1}, \ldots, T_{k-1}\right)$ with $0<T_{1}<T_{2}<\cdots<$ $T_{k-1}<1$ and having an absolutely continuous distribution on $[0,1]^{k-1}$.

Proof. The proof of (i) and (ii) (Case $k=3$ ) can be found in [9]. It suffices to prove (ii).

Below, for $1 \leq j \leq k-1, \tau_{j, t}$ will be denoted by $\tau_{j}$. It can be shown that it suffices to show that for any $1 \leq i_{1}<i_{2} \cdots<i_{p}<k$ and $0<r_{1}<r_{2}<\cdots<r_{p}<r_{p+1}<1=$ $r_{p+2}$,

$$
\lim _{t \rightarrow \infty} P\left(\frac{\tau_{i_{1}}}{t}<r_{1}<\frac{\tau_{i_{2}}}{t}<r_{2}<\cdots<\frac{\tau_{i_{p}}}{t}<r_{p}<\frac{\tau_{k-1}}{t}<r_{p+1}<1 \mid A_{t}\right)
$$

exists and is absolutely continuous.

Suppose that at time $t r_{1}$ there are $n_{1} \in \mathbb{N}$ particles of which $k_{1}$ have descendants that survive till time $t r_{2}$. For each $1 \leq j \leq k_{1}$, suppose there are $n_{2 j} \in \mathbb{N}$ descendants alive at time $t r_{2}$ and for each such $j$, let $k_{2 j}$ out of the $n_{2 j}$ have descendants that survive till time $t_{3}$. Let $\mathbf{k}_{\mathbf{2}}=\left(k_{21}, \ldots, k_{2 k_{1}}\right)$ and $\left|\mathbf{k}_{\mathbf{2}}\right|=\sum_{j=1}^{k_{1}} k_{2 j}$. Inductively, for $i=3, \ldots, p+1$, at time $t r_{i}$, there are $n_{i j}$ descendants for the $j$-th particle, $1 \leq j \leq\left|\mathbf{k}_{\mathbf{i}-\mathbf{1}}\right|$. For each such $j$, let $k_{i j}$ out of $n_{i j}$ have descendants that survive up till time $t r_{i+1}$ (see figure 1 for an illustration). 


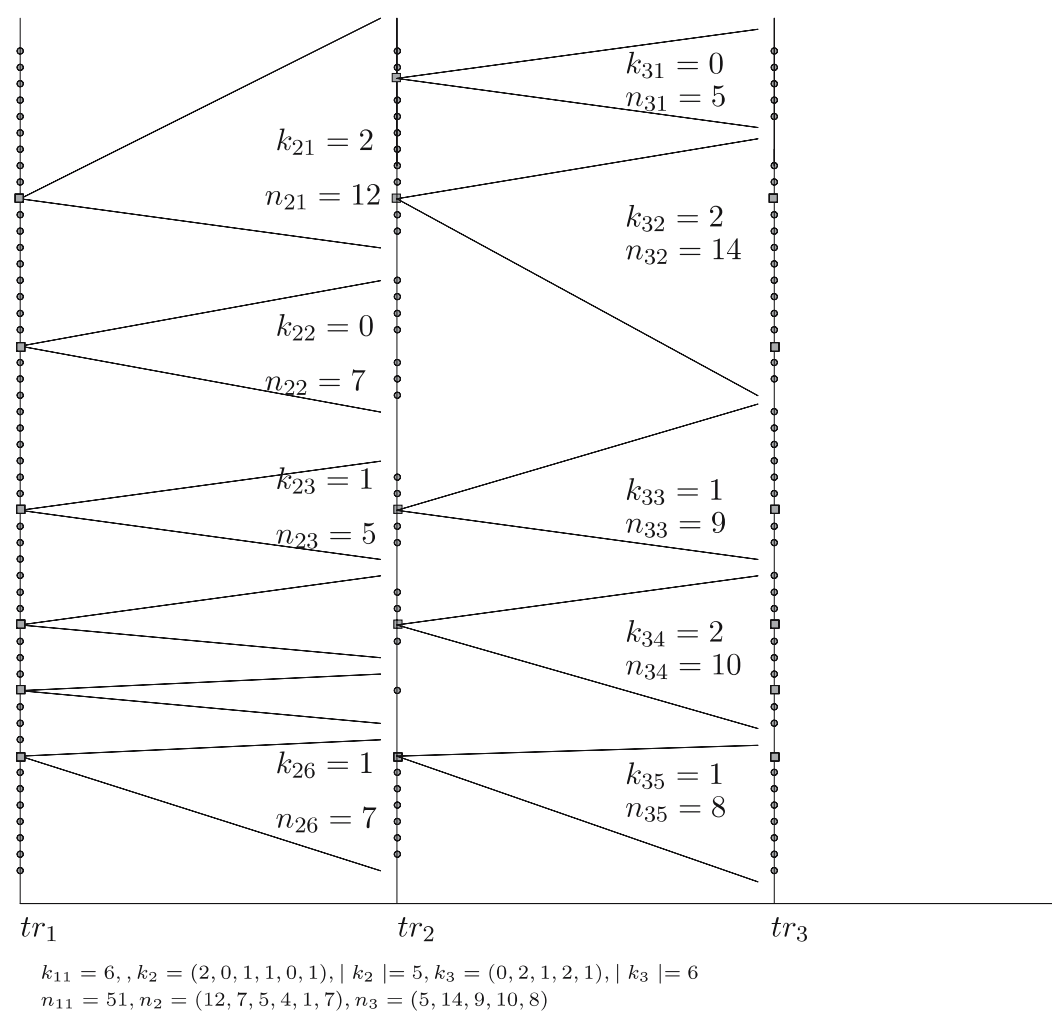

Figure 1. Tracking particles surviving at various times.

Let $\mathbb{N}_{1}=\left\{\left(n_{1}, k_{1}\right): n_{1}, k_{1} \in \mathbb{N}, k_{1} \leq n_{1}\right\}$. For $i=2, \ldots p+1$ let $\mathbf{n}_{\mathbf{i}}=\left(n_{i 1}, n_{i 2}\right.$, $\left.\ldots, n_{i\left|\mathbf{k}_{\mathbf{i}-1}\right|}\right), \mathbf{k}_{\mathbf{i}}=\left(k_{i 1}, k_{i 2}, \ldots, k_{i\left|\mathbf{k}_{\mathbf{i}-1}\right|}\right)$, such that $n_{i j}, k_{i j} \in \mathbb{N}$, and $\left|\mathbf{k}_{\mathbf{i}}\right| \equiv \sum_{j=1}^{\left|\mathbf{k}_{\mathbf{i}-1}\right|} k_{i j}$. Let $\mathbb{N}_{i}=\left\{\left(\mathbf{n}_{\mathbf{i}}, \mathbf{k}_{\mathbf{i}}\right): k_{i j} \leq n_{i j}\right.$ for $\left.2 \leq j \leq\left|\mathbf{k}_{\mathbf{i}-\mathbf{1}}\right|\right\}$ and $\hat{\mathbb{N}}=\prod_{i=1}^{p+1} \mathbb{N}_{i}$.

Let $f_{s} \equiv P\left(N_{s}>0 \mid N_{0}=1\right)$. Now,

$$
\begin{aligned}
P\left(\frac{\tau_{i_{1}}}{t}<r_{1}<\frac{\tau_{i_{2}}}{t}<r_{2}<\cdots<\frac{\tau_{i_{p}}}{t}<r_{p}<\frac{\tau_{k-1}}{t}<r_{p+1}<1 \mid A_{t}\right) \\
=\frac{f_{t r_{1}}}{f_{t}} \sum_{\hat{n} \in \hat{\mathbb{N}}}\left(\left(\begin{array}{l}
n_{1} \\
k_{1}
\end{array}\right)\left(f_{t u_{1}}\right)^{k_{1}}\left(1-f_{t u_{1}}\right)^{n_{1}-k_{1}}\right) P\left(N_{t r_{1}}=n_{1} \mid N_{t r_{1}}>0\right) \\
\quad \times \prod_{i=2}^{p+1\left|\mathbf{k}_{i-1}\right|}\left(\left(\begin{array}{l}
n_{i j} \\
k_{i j}
\end{array}\right)\left(f_{t u_{i}}\right)^{k_{i j}}\left(1-f_{t u_{i}}\right)^{n_{i j}-k_{i j}}\right) \\
\quad \times P\left(N_{t u_{i}}^{(j)}=n_{i j} \mid N_{t u_{i}}^{(j)}>0\right) g(\mathbf{k}) E\left(\frac{\prod_{j=1}^{k} X^{(j)}}{S^{k}}\right),
\end{aligned}
$$

with $u_{i}=r_{i+1}-r_{i}, i=1,2, \ldots, p+1, N_{t u_{i}}^{(j)}$ is the number of particles alive at time $t u_{i}$ of the age-dependent branching process starting with one particle namely $j$, $g(\mathbf{k})=g\left(k_{1}, \ldots, k_{p}\right)$ is the proportion of configurations that have the desired number of 
ancestors corresponding to the event of interest in (2.13), $X^{(j)} \stackrel{d}{=} N_{t u_{p+1}}^{(j)} \mid\left(N_{t u_{p+1}}^{(j)}>0\right)$ and $S=\sum_{j=1}^{\left|\mathbf{k}_{\mathbf{p}+\mathbf{1}}\right|} X^{(j)}$.

Let $q_{1}=\frac{r_{1}}{u_{1}}$ and $q_{i}=\frac{u_{i}}{u_{i+1}}$ for $2 \leq i \leq p+1$. Then following [9] and using Proposition 2.1(a) and (b) repeatedly we can show that $P\left(\frac{\tau_{i_{1}}}{t}<r_{1}<\frac{\tau_{i_{2}}}{t}<r_{2}<\cdots<\right.$ $\left.\frac{\tau_{i p}}{t}<r_{p}<\frac{\tau_{k-1}}{t}<r_{k-1}<1 \mid A_{t}\right)$ converges to

$$
\begin{aligned}
& \frac{1}{r_{1}} \sum_{k_{i} \in \mathbb{N}^{\left|\mathbf{k}_{\mathbf{i}-\mathbf{1}}\right|}} \int \mathrm{d} x \mathrm{e}^{-x}\left(q_{1} x\right)^{k_{1}} \frac{1}{k_{1} !} \mathrm{e}^{-x q_{1}} \\
& \quad \times \prod_{i=2}^{p+1} \prod_{j=1}^{\left|\mathbf{k}_{\mathbf{i}-\mathbf{1}}\right|} \int \mathrm{d} x \mathrm{e}^{-x} \frac{\left(q_{i} x\right)^{k_{i j}}}{k_{i j} !} \mathrm{e}^{-x q_{i}} \times g(\mathbf{k}) \\
& \quad \times \int \prod_{i=1}^{k+1} \mathrm{~d} x_{i}\left(\frac{\prod_{i=1}^{k} x_{i}}{\left(\sum_{i=1}^{k+1} x_{i}\right)^{k}}\right) \mathrm{e}^{-\sum_{i=1}^{k+1} x_{i}} \frac{\left(x_{k+1}\right)^{\left|\mathbf{k}_{\mathbf{p}+\mathbf{1}}\right|-(k+1)}}{\left(\left|\mathbf{k}_{\mathbf{p}+\mathbf{1}}\right|-(k+1)\right) !} \\
& =\frac{1}{r_{1}} \sum_{\mathbf{k}_{\mathbf{i}} \in \mathbb{N}^{\left|\mathbf{k}_{\mathbf{i}} \mathbf{1}\right|}} \prod_{i=1}^{p+1} \frac{\left(q_{i}\right)^{\left|\mathbf{k}_{\mathbf{i}}\right|}}{\left(1+q_{i}\right)^{\left|\mathbf{k}_{\mathbf{i}}\right|-\left|k_{i-1}\right|} g(\mathbf{k})} \\
& \quad \times \int \prod_{i=1}^{k+1} \mathrm{~d} x_{i}\left(\frac{\prod_{i=1}^{k} x_{i}}{\left(\sum_{i=1}^{k+1} x_{i}\right)^{k}}\right) \mathrm{e}^{-\sum_{i=1}^{k+1} x_{i}} \frac{\left(x_{k+1}\right)^{\left|\mathbf{k}_{\mathbf{p}+\mathbf{1}}\right|-(k+1)}}{\left(\left|\mathbf{k}_{\mathbf{p}+\mathbf{1}}\right|-(k+1)\right) !} .
\end{aligned}
$$

Consequently, we have shown that the random vector $\tilde{\tau}_{t}$ converges in distribution to a random vector $\tilde{T}$. Since the right-hand side of (2.14) is a differentiable function of $\left(r_{1}, r_{2}, \ldots, r_{k-1}\right)$, it follows that $\tilde{T}$ has an absolutely continuous distribution on $[0,1]^{k-1}$.

\section{Proof of Theorem 1.1}

Using Notation 1.1 we have

$$
a_{t}=t-\sum_{i=1}^{M_{t}} L_{t i},
$$

whenever $M_{t}>0$ and is equal to $a+t$ otherwise; and that

$$
X_{t}=X_{0}+\sum_{i=1}^{M_{t}} \eta_{t i}\left(L_{t i}\right)+\eta_{t\left(M_{t}+1\right)}\left(a_{t}\right) .
$$

Rearranging the terms, we obtain

$$
\begin{aligned}
& \left(a_{t}, \frac{X_{t}}{\sqrt{t}}\right) \\
& \quad=\left(a_{t}, \sqrt{\frac{1}{\mu}} H_{t}\right)+\left(0,\left(\sqrt{\frac{M_{t}}{t}}-\sqrt{\frac{1}{\mu}}\right) H_{t}\right)+\left(0, J_{t}\right)+\left(0, \frac{X_{0}}{\sqrt{t}}\right),
\end{aligned}
$$


where $H_{t}=\frac{\sum_{i=1}^{M_{t}} \eta_{t i}\left(L_{t_{i}}\right)}{\sqrt{M_{t}}}$ and $J_{t}=\frac{1}{\sqrt{t}} \eta_{t\left(M_{t}+1\right)}\left(a_{t}\right)$. We shall show that as $t \rightarrow \infty$,

$$
J_{t} \mid A_{t} \stackrel{p}{\rightarrow} 0
$$

and

$$
\left(a_{t}, \frac{1}{\sqrt{\mu}} H_{t}\right) \mid A_{t} \stackrel{d}{\longrightarrow}(U, V) .
$$

As $\frac{X_{0}}{\sqrt{t}} \stackrel{p}{\rightarrow} 0$, an application of Proposition 2.1(c) along with Slutsky's theorem will yield the result. So we now prove (3.1) and (3.2).

Proof of (3.1). Let $\epsilon>0$ be given.

$$
\begin{aligned}
P\left(\left|J_{t}\right|>\epsilon \mid A_{t}\right) & \leq P\left(\left|J_{t}\right|>\epsilon, a_{t} \leq k \mid A_{t}\right)+P\left(\left|J_{t}\right|>\epsilon, a_{t}>k \mid A_{t}\right) \\
& \leq P\left(\left|J_{t}\right|>\epsilon, a_{t} \leq k \mid A_{t}\right)+P\left(a_{t}>k \mid A_{t}\right) \\
& \leq \frac{E\left(\left|J_{t}\right|^{2} I_{a_{t} \leq k} \mid A_{t}\right)}{\epsilon^{2}}+P\left(a_{t}>k \mid A_{t}\right) .
\end{aligned}
$$

By Proposition 2.1 and the ensuing tightness, for any $\eta>0$ there is a $k_{\eta}$ such that

$$
P\left(a_{t}>k \mid A_{t}\right)<\frac{\eta}{2}
$$

for all $k \geq k_{\eta}, t \geq 0$. Next,

$$
\begin{aligned}
E\left(\left|J_{t}\right|^{2} I_{a_{t} \leq k_{\eta}} \mid A_{t}\right) & =E\left(I_{a_{t} \leq k_{\eta}} E\left(\left|J_{t}\right|^{2} \mid \mathcal{L}_{t}\right) \mid A_{t}\right) \\
& =E\left(I_{a_{t} \leq k_{\eta}} \frac{v\left(a_{t}\right)}{t} \mid A_{t}\right) \\
& \leq \frac{\sup _{u \leq k_{\eta}} v(u)}{t} .
\end{aligned}
$$

Hence,

$$
P\left(\left|J_{t}\right|>\epsilon \mid A_{t}\right) \leq \frac{\sup _{u \leq k_{\eta}} v(u)}{t \epsilon^{2}}+\frac{\eta}{2} .
$$

Since $\epsilon>0$ and $\eta>0$ are arbitrary this proves (3.1).

Proof of (3.2). Now we consider the second term. For $\lambda>0, \theta \in \mathbb{R}$, as $a_{t}$ is $\mathcal{L}_{t}$ measurable we have

$$
\begin{aligned}
E\left(\mathrm{e}^{-\lambda a_{t}} \mathrm{e}^{-i \frac{\theta}{\sqrt{\mu}} H_{t}} \mid A_{t}\right)= & E\left(\mathrm{e}^{-\lambda a_{t}}\left(E\left(\mathrm{e}^{-i \theta H_{t}} \mid \mathcal{L}_{t}\right)-\mathrm{e}^{-\frac{\theta^{2} \psi}{2 \mu}}\right) \mid A_{t}\right) \\
& +\mathrm{e}^{-\frac{\theta^{2} \psi}{2 \mu}} E\left(\mathrm{e}^{-\lambda a_{t}} \mid A_{t}\right) .
\end{aligned}
$$

Proposition 2.4 shows that the first term above converges to zero and using Proposition 2.1(d), (3.2) is immediate. 


\section{Proof of Theorem 1.2}

Let $\phi \in C_{b}^{+}\left(\mathbb{R} \times \mathbb{R}_{+}\right)$. We shall show, for each $k \geq 1$, that the moment-functions $E\left(\frac{\left\langle\phi, \tilde{Y}_{t}\right\rangle^{k}}{N_{t}^{k}} \mid A_{t}\right)$ converge as $t \rightarrow \infty$. Then, by Theorem 16.16 in [14] the result follows.

The case $k=1$ follows from Theorem 1.1 and the bounded convergence theorem. We shall next consider the case $k=2$. Pick two individuals $C_{1}, C_{2}$ at random (i.e. by simple random sampling without replacement) from those alive at time $t$. Let the age and position of the two individuals be denoted by $\left(a_{t}^{i}, X_{t}^{i}\right), i=1,2$.

$$
\begin{aligned}
E( & \left.\left(\frac{\left\langle\phi, \tilde{Y}_{t}\right\rangle}{N_{t}}\right)^{2} \mid A_{t}\right) \\
= & E\left(\frac{\sum_{i=1}^{N_{t}} \phi^{2}\left(a_{t}^{i}, \frac{X_{t}^{i}}{\sqrt{t}}\right)+\sum_{\substack{i, j=1 \\
i \neq j}}^{N_{t}} \phi\left(a_{t}^{i}, \frac{X_{t}^{i}}{\sqrt{t}}\right) \phi\left(a_{t}^{j}, \frac{X_{t}^{j}}{\sqrt{t}}\right)}{N_{t}^{2}} \mid A_{t}\right) \\
= & E\left(\frac{1}{N_{t}^{2}} \sum_{i=1}^{N_{t}} \phi^{2}\left(a_{t}^{i}, \frac{X_{t}^{i}}{\sqrt{t}}\right) \mid A_{t}\right) \\
& +E\left(\frac{1}{N_{t}^{2}} \sum_{\substack{i, j=1 \\
i \neq j}}^{N_{t}}\left(a_{t}^{i}, \frac{X_{t}^{i}}{\sqrt{t}}\right) \phi\left(a_{t}^{j}, \frac{X_{t}^{j}}{\sqrt{t}}\right) \mid A_{t}\right) \\
= & E\left(\frac{1}{N_{t}} E\left(\phi^{2}\left(a_{t}^{1}, \frac{X_{t}^{1}}{\sqrt{t}}\right) \mid N_{t}\right) \mid A_{t}\right) \\
& +E\left(\frac{N_{t}\left(N_{t}-1\right)}{N_{t}^{2}} E\left(\phi\left(a_{t}^{1}, \frac{X_{t}^{1}}{\sqrt{t}}\right) \phi\left(a_{t}^{2}, \frac{X_{t}^{2}}{\sqrt{t}}\right) \mid N_{t}\right) \mid A_{t}\right) \\
= & E\left(\frac{1}{N_{t}} E\left(\phi^{2}\left(a_{t}^{1}, X_{t}^{1}\right)-\phi\left(a_{t}^{1}, \frac{X_{t}^{1}}{\sqrt{t}}\right) \phi\left(a_{t}^{2}, \frac{X_{t}^{2}}{\sqrt{t}}\right) \mid N_{t}\right) \mid A_{t}\right) \\
& +E\left(E\left(\phi^{2}\left(a_{t}^{1}, X_{t}^{1}\right)-\phi\left(a_{t}^{1}, \frac{X_{t}^{1}}{\sqrt{t}}\right) \phi\left(a_{t}^{2}, \frac{X_{t}^{2}}{\sqrt{t}}\right) \mid N_{t}\right) \mid A_{t}\right) \\
& \left.\left.+E\left(a_{t}^{2}, \frac{X_{t}^{2}}{\sqrt{t}}\right) \mid N_{t}\right) \mid A_{t}\right) \\
\sqrt{t}) & \\
= & \left.\left(a_{t}^{2}, \frac{X_{t}^{2}}{\sqrt{t}}\right) \mid A_{t}\right) .
\end{aligned}
$$

As $\phi$ is bounded, the first term on the right hand side in (4.1) is bounded above by a constant times $E\left(\frac{1}{N_{t}} \mid A_{t}\right)$, which goes to 0 as $t \rightarrow \infty$ by Proposition 2.1(b). We will now analyse the second term in (4.1). Let $\tau_{t}=\tau_{C_{1}, C_{2}, t}$ be the death time of the common ancestor, say $D$, of $C_{1}, C_{2}$. Let the position of $D$ at time $\tau_{t}$ be given by $\tilde{X}_{\tau_{t}}$. Let the net displacement of $C_{1}$ and $C_{2}$ from $D$ be denoted by $X_{t-\tau_{t}}^{i}, i=1,2$ respectively. Then $X_{t}^{i}=\tilde{X}_{\tau_{t}}+X_{t-\tau_{t}}^{i}, i=1,2$. 
Conditioned on the history up to the death of $D$, say $\mathcal{G}_{t}$, the random variables $\left(a_{t}^{i}, X_{t-\tau_{t}}^{i}\right), i=1,2$ are independent. By Theorem 2.1(i) conditioned on $A_{t}, \frac{\tau_{t}}{t}$ converges in distribution to an absolutely continuous random variable $T$ (say) in $[0,1]$. Also by Theorem 1.1 conditioned on $\mathcal{G}_{t}$ and $A_{t},\left\{\left(a_{t}^{i}, \frac{X_{t-\tau_{t}}^{i}}{\sqrt{t-\tau_{t}}}\right), i=1,2\right\}$ converges in distribution to $\left\{\left(U_{i}, V_{i}\right), i=1,2\right\}$ which are i.i.d. with distribution $(U, V)$ as in Theorem 1.1. Further, using Theorem 1.1 again, $\frac{\tilde{X}_{\tau_{t}}}{\sqrt{\tau_{t}}}$ conditioned on $A_{\tau_{t}}$ converges in distribution to a random variable $S$ distributed as $V$ and independent of $\left\{\left(U_{i}, V_{i}\right), i=1,2\right\}$.

Combining these one can conclude that $\left\{\left(a_{t}^{i}, \frac{X_{t}^{i}}{\sqrt{t}}\right), i=1,2\right\}$ conditioned on $A_{t}$ converges in distribution to $\left\{\left(U_{i}, \sqrt{T} S+\sqrt{(1-T)} V_{i}\right), i=1,2\right\}$. Thus for any $\phi \in C_{b}\left(\mathbb{R}_{+} \times \mathbb{R}\right)$ we have, by the bounded convergence theorem,

$$
\begin{aligned}
& \lim _{t \rightarrow \infty} E\left(\prod_{i=1}^{2} \phi\left(a_{t}^{i}, \frac{X_{t}^{i}}{\sqrt{t}}\right) \mid A_{t}\right) \\
& =E \prod_{i=1}^{2} \phi\left(U_{i}, \sqrt{T} S+\sqrt{(1-T)} V_{i}\right) \equiv m_{2}(\phi) \quad \text { (say). }
\end{aligned}
$$

Hence we have shown that the second term of (4.1) converges to $m_{2}(\phi)$ and we are done.

Next, we consider the case $k>2$. For any $\phi \in C_{b}^{+}\left(\mathbb{R} \times \mathbb{R}_{+}\right)$one has

$$
\begin{aligned}
E\left(\frac{\left\langle\phi, \tilde{Y}_{t}\right\rangle^{k}}{N_{t}^{k}} \mid A_{t}\right)= & E\left(\frac{N_{t}\left(N_{t}-1\right)\left(N_{t}-2\right) \cdots\left(N_{t}-k\right)}{N_{t}^{k}} \sum_{\mathbf{i} \in C_{k}} \prod_{j=1}^{k} \phi\left(a_{t}^{i_{j}}, \frac{X_{t}^{i_{j}}}{\sqrt{t}}\right) \mid A_{t}\right) \\
& +E\left(O\left(\frac{1}{N_{t}}\right) \mid A_{t}\right),
\end{aligned}
$$

where $C_{k}$ is the collection of indices of the $k$ particles sampled without replacement from the particles alive at time $t$. We shall deem $C_{k}=$ on the event $\left\{N_{t}<k\right\}$. Consider one such sample, $\mathbf{i}$, and trace the genealogical tree $T \in \mathcal{T}_{\mathbf{i}}(k),\left(\mathcal{T}_{\mathbf{i}}(k)\right.$ is the collection of all possible labelled trees with $k$ leaves given by $\mathbf{i}$ ), until their most recent common ancestor. For any leaf $i_{j}$ in $T$, let $1=n\left(i_{j}, 1\right)<n\left(i_{j}, 2\right)<\cdots<n\left(i_{j}, N_{i_{j}}\right)$ be the labels of the internal nodes on the path from leaf $i_{j}$ to the root. We list the ancestoral death times on this by $\left\{\tau_{1}, \tau_{n\left(i_{j}, 1\right)}, \ldots, \tau_{n\left(i_{j}, N_{i_{j}}\right)}\right\}$. Finally, we denote the net displacement of the ancestors in the time intervals

$$
\left[0, \tau_{1}\right],\left[\tau_{1}, \tau_{n\left(i_{j}, 2\right)}\right], \ldots,\left[\tau_{n\left(i_{j}, N_{i_{j}}-1\right)}, \tau_{n\left(i_{j}, N_{i_{j}}\right)}\right],\left[\tau_{n\left(i_{j}, N_{i_{j}}\right)}, t\right]
$$

by

$$
\tilde{\eta}_{i_{j}}^{1}\left(\tau_{1}\right), \tilde{\eta}_{i_{j}}^{2}\left(\tau_{n\left(i_{j}, 2\right)}, \tau_{1}\right), \ldots, \tilde{\eta}_{i_{j}}^{N_{i_{j}}}\left(\tau_{n\left(i_{j}, N_{i_{j}}\right)}, \tau_{n\left(i_{j}, N_{i_{j}}-1\right)}\right), \tilde{\eta}_{i_{j}}^{\prime}\left(t, \tau_{n\left(i_{j}, N_{i_{j}}\right)}\right) .
$$

Given the above notation we have

$$
\begin{aligned}
E\left(\prod_{j=1}^{k} \phi\left(a_{t}^{i_{j}}, \frac{X_{t}^{i_{j}}}{\sqrt{t}}\right) \mid A_{t}\right)= & E\left(\sum _ { T \in \mathcal { T } _ { i } } \prod _ { j = 1 } ^ { k } \phi \left(a_{t}^{i_{j}}, \frac{1}{\sqrt{t}}\left(\tilde{\eta}_{i_{j}}^{1}\left(\tau_{1}\right)\right.\right.\right. \\
& \left.+\sum_{m=2}^{N_{i_{j}}} \tilde{\eta}_{i_{j}}^{m}\left(\tau_{n\left(i_{j}, m\right)}, \tau_{n\left(i_{j}, m-1\right)}\right)+\tilde{\eta}_{i_{j}}^{\prime}\left(t, \tau_{n\left(i_{j}, N_{i_{j}}\right)}\right) \mid A_{t}\right) .
\end{aligned}
$$


Now by Theorem 2.1,

$$
\frac{\left(\tau_{1}, \tau_{n\left(i_{j}, 2\right)}, \ldots, \tau_{n\left(i_{j}, N_{i_{j}}\right)}\right)}{t} \mid A_{t} \stackrel{d}{\longrightarrow}\left(T_{1}, T_{n\left(i_{j}, 2\right)}, \ldots, T_{n\left(i_{j}, N_{i_{j}}\right)}\right) .
$$

Further using Theorem 1.1 repeatedly, with appropriate conditioning as in the case $k=2$ we obtain

$$
\begin{aligned}
\lim _{t \rightarrow \infty} E\left(\frac{\left\langle\phi, \tilde{Y}_{t}\right\rangle^{k}}{N_{t}^{k}} \mid A_{t}\right)= & E\left(\sum _ { i \in C _ { k } } \sum _ { T \in \mathcal { T } _ { \mathbf { i } } } \prod _ { j = 1 } ^ { k } \phi \left(U_{i_{j}}, S_{i_{j}} \sqrt{T_{1}}\right.\right. \\
& \left.\left.+\sum_{m=2}^{N_{i_{j}}} Z_{i_{j}}^{m} \sqrt{T_{n\left(i_{j}, m\right)}-T_{n\left(i_{j}, m-1\right)}}+Z_{i_{j}}^{\prime} \sqrt{1-T_{n\left(i_{j}, N_{i_{j}}\right)}}\right) \mid A_{t}\right) \\
\equiv & m_{k}(\phi),
\end{aligned}
$$

where $S_{i_{j}}, Z_{i_{j}}^{\prime}, Z_{i_{j}}^{m}, m=2, \ldots, N_{i_{j}}$ are i.i.d. random variables distributed as $V$, and $U_{i_{j}}$ are independent random variables distributed as $U$. For $\phi \in C_{b}(\mathbb{R})$, the sequence $\left\{m_{k}(\phi): k \geq 1\right\}$ is necessarily a moment sequence of a unique probability distribution on $\mathbb{R}$. This being true for each $\phi$, by Theorem 16.16 in [14] we are done.

\section{Proof of Theorem 1.3}

As stated in the theorem we scale the age and motion parameters differently, to obtain a super-process limit. Theorem 1.1 is used in establishing the limiting log-Laplace equation (Propositions 5.2 and 5.3). Tightness of the underlying particle system is shown in Proposition 5.4 and the result then follows by the standard techniques (see [7]).

Let $C_{b}\left(\mathbb{R}_{+} \times \mathbb{R}\right)$ be the set of bounded continuous function on $\mathbb{R}_{+} \times \mathbb{R}$ and $C_{b}^{1,2}\left(\mathbb{R}_{+} \times \mathbb{R}\right)$ be the set of bounded functions which are differentiable in the first variable and are twice differentiable in the second variable. Let $Z$ be the branching Markov process $Y$ described earlier, with $Y_{0}=\delta_{(a, x)}$, lifetime $G$ exponential with mean $\lambda, p_{1}=1$ and $\eta \stackrel{d}{=} \eta_{1}$ (see (1.10)). Note that $Z_{t}$ has the representation given by (1.3)-(1.5) with $X_{0}=x$. For any $\phi \in C_{b}\left(\mathbb{R}_{+} \times \mathbb{R}\right)$, define the semigroup

$$
S_{t} \phi(a, x) \equiv E_{(a, x)}\left[\left\langle Z_{t}, \phi\right\rangle\right]=E_{(a, x)}\left[\phi\left(a_{t}, X_{t}\right)\right] .
$$

Conditioning on the first birth we obtain the following evolution equation for $S_{t}$ :

$$
S_{t} \phi(a, x)=\mathrm{e}^{-\lambda t} T_{t} \phi(a, x)+\int_{0}^{t} \mathrm{~d} s \lambda \mathrm{e}^{-\lambda s} T_{s}\left(S_{t-s}(\phi)(0, \cdot)\right)(a, x),
$$

where $T_{t}$ is the semigroup associated to $\mathcal{L}$ given by

$$
\mathcal{L} f(a, x)=\frac{\partial f}{\partial a}(a, x)+\frac{\sigma^{2}(a)}{2} \Delta f(a, x),
$$


with $f \in C_{b}^{1,2}\left(\mathbb{R}_{+} \times \mathbb{R}\right)$ and $\Delta$ being the one-dimensional Laplacian. Making a change of variable $s \rightarrow t-s$ in the second term of (5.1) and then differentiating it with respect to $t$, we have

$$
\begin{aligned}
\frac{\mathrm{d}}{\mathrm{d} t} S_{t}(\phi)(a, x)= & -\lambda \mathrm{e}^{-\lambda t} T_{t} \phi(a, x)+\mathrm{e}^{-\lambda t} \mathcal{L} T_{t} \phi(a, x)+\lambda S_{t}(\phi)(0, x) \\
& +\int_{0}^{t} \mathrm{~d} s \lambda\left(-\lambda \mathrm{e}^{-\lambda(t-s)}\right) T_{t-s}\left(S_{s}(\phi)(0, \cdot)\right)(a, x) \\
& +\int_{0}^{t} \mathrm{~d} s \lambda \mathrm{e}^{-\lambda(t-s)} \mathcal{L} T_{t-s}\left(S_{s}(\phi)(0, \cdot)\right)(a, x) \\
= & \lambda S_{t}(\phi)(0, x)+(\mathcal{L}-\lambda)\left[\mathrm{e}^{-\lambda t} T_{t} \phi(a, x)\right. \\
& \left.+\int_{0}^{t} \mathrm{~d} s \lambda \mathrm{e}^{-\lambda(t-s)} T_{t-s}\left(S_{S}(\phi)(0, \cdot)\right)(a, x)\right] \\
= & \lambda S_{t}(\phi)(0, x)+(\mathcal{L}-\lambda) S_{t}(\phi)(a, x) \\
= & \frac{\partial S_{t} \phi}{\partial a}(a, x)+\frac{\sigma^{2}(a)}{2} \Delta S_{t} \phi(a, x)+\lambda\left(S_{t}(\phi)(0, x)-S_{t}(\phi)(a, x)\right) .
\end{aligned}
$$

Hence the generator of $S_{t}$ is given by

$$
\mathcal{R}_{t} f(a, x)=\mathcal{L} f(a, x)+\lambda(f(0, x)-f(a, x)), \quad f \in C_{b}^{1,2}\left(\mathbb{R}_{+} \times \mathbb{R}\right) .
$$

For each $n \geq 1$, define (another semigroup)

$$
R_{t}^{n} \phi(a, x) \equiv E_{a, 0}\left(\phi\left(a_{t}, x+\frac{X_{t}}{\sqrt{n}}\right)\right), \quad \phi \in C_{b}\left(\mathbb{R}_{+} \times \mathbb{R}\right) .
$$

Now using the representation (1.3)-(1.4) and (1.10), it is easily seen that the semigroup $R_{t}^{n}$ is the semigroup $S_{t}$ with $\sigma$ replaced by $\frac{\sigma}{\sqrt{n}}$. Hence, from (5.2), (5.3) it follows that the generator of the semigroup $R_{t}^{n}$ will be given by

$$
\mathcal{R}^{n} f(a, x)=\mathcal{L}^{n} f(a, x)+\lambda(f(0, x)-f(a, x)),
$$

where

$$
\mathcal{L}^{n} f(a, x)=\frac{\partial f}{\partial a}(a, x)+\frac{\sigma^{2}(a)}{2 n} \Delta f(a, x), \quad f \in C_{b}^{1,2}\left(\mathbb{R}_{+} \times \mathbb{R}\right) .
$$

\section{PROPOSITION 5.1}

Let $\epsilon>0$ and $t \geq \epsilon$. Let $\phi \in C_{l}^{+}\left(\mathbb{R}_{+} \times \mathbb{R}\right)$. Then,

$$
\sup _{(a, x) \in \mathbb{R}_{+} \times \mathbb{R}}\left|R_{n t}^{n}(\phi)(a, x)-U_{t}(\phi)(x)\right| \rightarrow 0 .
$$

Proof. Let $t \geq \epsilon$. Applying Theorem 1.1 to the process $Z$, we have $\left(a_{n t}, \frac{X_{n t}}{\sqrt{n}}\right) \stackrel{d}{\longrightarrow}(U, t V)$. The proposition is then immediate from the bounded convergence theorem and the fact that $\phi \in C_{l}^{+}\left(\mathbb{R}_{+} \times \mathbb{R}\right)$. 


\section{PROPOSITION 5.2}

Let $\pi_{n v}$ be as in Notation 1.2. The log-Laplace functional of $\mathcal{Y}_{t}^{n}$ is given by

$$
E_{\pi_{n v}}\left[\mathrm{e}^{-\left\langle\phi, \mathcal{Y}_{t}^{n}\right\rangle}\right]=\mathrm{e}^{-\left\langle u_{t}^{n} \phi, v\right\rangle}
$$

where

$$
u_{t}^{n} \phi(a, x)=R_{n t}^{n} n\left(1-\mathrm{e}^{-\frac{\phi}{n}}\right)(a, x)-\lambda \int_{0}^{t} \mathrm{~d} s R_{n(t-s)}^{n}\left(n^{2} \Psi_{n}\left(\frac{u_{s}^{n} \phi}{n}\right)\right)(a, x)
$$

where

$$
\Psi_{n}(\phi)(a, x):=\left[F_{n}(1-\phi(0, x))-(1-\phi(0, x))\right] .
$$

Proof. For any $n \in \mathbb{N}$, let $Y_{t}^{n}$ be the sequence of branching Markov processes defined in $\S 1.2$. It can be shown that its $\log$-Laplace functional $L_{t}^{n}$ satisfies

$$
L_{n t}^{n} \phi(a, x)=\mathrm{e}^{-\lambda n t} T_{n t}^{n}\left[\mathrm{e}^{-\phi}\right](a, x)+\int_{0}^{n t} \mathrm{~d} s \lambda \mathrm{e}^{-\lambda s} T_{s}^{n}\left[F_{n}\left(L_{n t-s}^{n} \phi(0, \cdot)\right)\right](a, x),
$$

where $t \geq 0$ and $T_{t}^{n}$ is the semigroup associated with $\mathcal{L}^{n}$. Using the fact that $\mathrm{e}^{-\lambda u}=$ $1-\int_{0}^{u} \mathrm{~d} s \lambda \mathrm{e}^{-\lambda s}$ for all $u \geq 0$ and a routine simplification, as done in [10], will imply that

$$
L_{n t}^{n} \phi(a, x)=T_{n t}^{n}\left[\mathrm{e}^{-\phi}\right](a, x)+\lambda \int_{0}^{n t} T_{n t-s}^{n}\left(F_{n}\left(L_{s}^{n} \phi(0, \cdot)\right)-L_{s}^{n} \phi\right)(a, x) \mathrm{d} s
$$

Therefore $v_{t}^{n}(\phi)(a, x)=1-L_{t}^{n} \phi(a, x)$, satisfies

$$
\begin{aligned}
& v_{n t}^{n} \phi(a, x) \\
& \left.\quad=T_{n t}^{n}\left(1-\mathrm{e}^{-\phi}\right)(a, x)+\int_{0}^{n t} \mathrm{~d} s T_{n t-s}^{n}\left(\left(1-v_{s}^{n} \phi\right)-F_{n}\left(1-v_{s}^{n}\right) \phi(0, \cdot)\right)\right)(a, x) \lambda .
\end{aligned}
$$

Then for $0 \leq s<t$,

$$
\begin{aligned}
\frac{\mathrm{d}}{\mathrm{d} s} R_{n(t-s)}^{n}\left(v_{n s}^{n}(\phi)\right)(a, x) \\
=-\left(n \mathcal{R}^{n}\right) R_{n(t-s)}^{n}\left(v_{n s}^{n}(\phi)\right)(a, x)+R_{n(t-s)}^{n}\left(\frac{\partial}{\partial s} v_{n s}^{n}(\phi)\right)(a, x) \\
=-\left(n \mathcal{R}^{n}\right) R_{n(t-s)}^{n}\left(v_{n s}^{n}(\phi)\right)(a, x) \\
\left.\quad+R_{n(t-s)}^{n}\left(n \mathcal{L}^{n} T_{n s}^{n}\left(1-e^{-\phi}\right)+n \lambda\left(\left(1-v_{n s}^{n} \phi\right)-F_{n}\left(1-v_{n s}^{n}\right) \phi(0, \cdot)\right)\right)(a, x)\right) \\
\quad+R_{n(t-s)}^{n}\left(\int_{0}^{n s} \mathrm{~d} r n \mathcal{L}^{n}\left(T_{n s-r}^{n}\left(\left(1-v_{r}^{n}(\phi)\right)-F_{n}\left(1-v_{r}^{n}\right) \phi(0, \cdot)\right)\right)(a, x)\right. \\
\left.=R_{n(t-s)}^{n} n\left(-\lambda\left(v_{n s}^{n}(\phi)(0, \cdot)-v_{n s}^{n}(\phi)\right)+\lambda\left(\left(1-v_{n s}^{n} \phi\right)-F_{n}\left(1-v_{n s}^{n}\right) \phi(0, \cdot)\right)\right)(a, x)\right) \\
=-R_{n(t-s)}^{n}\left(n \Psi_{n}\left(v_{n s}^{n} \phi\right)\right)(a, x) .
\end{aligned}
$$


Integrating both sides with respect to $s$ from 0 to $t$, we obtain that

$$
v_{n t}^{n}(\phi)(a, x)=R_{n t}^{n}\left(1-\mathrm{e}^{-\phi}\right)(a, x)-\int_{0}^{t} \mathrm{~d} s R_{n(t-s)}^{n}\left(n \Psi_{n}\left(v_{n s}^{n} \phi\right)\right)(a, x) .
$$

If $\pi_{n v}$ is a Poisson random measure with intensity $n v$, then

$$
E_{\pi_{n v}}\left[\mathrm{e}^{-\left\langle\phi, \mathcal{Y}_{t}^{n}\right\rangle}\right]=E_{\pi_{n v}}\left[\mathrm{e}^{-\left\langle\frac{\phi}{n}, Y_{n t}^{n}\right\rangle}\right]=\mathrm{e}^{\left\langle L_{t}^{n}\left(\frac{\phi}{n}\right)-1, n \nu\right\rangle}=\mathrm{e}^{-\left\langle n v_{t}^{n}\left(\frac{\phi}{n}\right), v\right\rangle} .
$$

Set $u_{t}^{n}(\phi) \equiv n v_{n t}^{n}\left(\frac{\phi}{n}\right)$. From (5.12), it is easy to see that $u_{t}^{n}(\phi)$ satisfies (5.7).

For any $f: \mathbb{R}_{+} \times \mathbb{R} \rightarrow \mathbb{R}$, we let $\|f\|_{\infty}=\sup _{(a, x) \in \mathbb{R}_{+} \times \mathbb{R}}|f(a, x)|$. With a little abuse of notation we shall let $\|f\|_{\infty}=\sup _{x \in \mathbb{R}}|f(x)|$ when $f: \mathbb{R} \rightarrow \mathbb{R}$ as well.

\section{PROPOSITION 5.3}

Let $\epsilon>0$. Let $\phi \in C_{l}^{+}\left(\mathbb{R}_{+} \times \mathbb{R}\right)$ (from Definition 1.1) and $u_{t}^{n}(\phi)$ be as in Proposition 5.2 and $u_{t}(\phi)$ be as in Theorem 1.3. Then for $t \geq \epsilon$,

$$
\sup _{(a, x) \in \mathbb{R}_{+} \times \mathbb{R}}\left|u_{t}^{n}(\phi)(a, x)-u_{t}(\phi)(x)\right| \rightarrow 0 .
$$

Proof. For any real $u \in \mathbb{R}$, define $\varepsilon_{n}(u)=\lambda n^{2}\left(F_{n}\left(1-\frac{u}{n}\right)-\left(1-\frac{u}{n}\right)\right)-\lambda c u^{2}$. So,

$$
\begin{aligned}
u_{t}^{n}(\phi)(a, x) & \\
= & R_{n t}^{n} n\left(1-\mathrm{e}^{-\frac{\phi}{n}}\right)(a, x)-\lambda \int_{0}^{t} \mathrm{~d} s R_{n(t-s)}^{n}\left(n^{2} \Psi_{n}\left(\frac{u_{s}^{n} \phi}{n}\right)\right)(a, x) \\
= & R_{n t}^{n} n\left(1-\mathrm{e}^{-\frac{\phi}{n}}\right)(a, x) \\
& -\int_{0}^{t} \mathrm{~d} s R_{n(t-s)}^{n}\left(\varepsilon_{n}\left(u_{s}^{n}(\phi(0 \cdot))\right)\right)(a, x) \\
& -\lambda c \int_{0}^{t} \mathrm{~d} s R_{n(t-s)}^{n}\left(u_{s}^{n} \phi(0, \cdot)^{2}\right)(a, x) .
\end{aligned}
$$

Now

$$
\begin{aligned}
u^{n} t(\phi)(a, x)-u_{t}(\phi)(x) & \\
= & R_{n t}^{n}\left(n\left(1-\mathrm{e}^{-\frac{\phi}{n}}\right)\right)(a, x)-U_{t}(\phi)(x) \\
& -\int_{0}^{t} \mathrm{~d} s R_{n(t-s)}^{n}\left(\varepsilon_{n}\left(u_{s}^{n}(\phi(0, \cdot))\right)\right)(a, x) \\
& +\lambda c \int_{0}^{t} \mathrm{~d} s\left(U_{t-s}\left(\left(u_{s} \phi\right)^{2}\right)(a, x)-R_{n(t-s)}^{n}\left(u_{s}^{n} \phi(0, \cdot)^{2}\right)(a, x)\right) \\
= & R_{n t}^{n}\left(n\left(1-\mathrm{e}^{-\frac{\phi}{n}}\right)\right)(a, x)-U_{t}(\phi)(x) \\
& -\int_{0}^{t} \mathrm{~d} s R_{n(t-s)}^{n}\left(\varepsilon_{n}\left(u_{s}^{n}(\phi(0, \cdot))\right)\right)(a, x)
\end{aligned}
$$




$$
\begin{aligned}
& +\lambda c \int_{0}^{t} \mathrm{~d} s R_{n(t-s)}^{n}\left(\left(u_{s} \phi\right)^{2}-u_{s}^{n} \phi(0, \cdot)^{2}\right)(a, x) \\
& \left.+\lambda c \int_{0}^{t} \mathrm{~d} s\left(U_{t-s}\left(\left(u_{s} \phi\right)^{2}\right)(x)-R_{n(t-s)}^{n}\left(u_{s} \phi\right)^{2}\right)(a, x)\right) .
\end{aligned}
$$

Observe that $R^{n}$ is a contraction, $\left\|u_{.}^{n}(\phi)\right\|_{\infty} \leq\|\phi\|_{\infty}$ and $\|u .(\phi)\|_{\infty} \leq\|\phi\|_{\infty}$ for $\phi \in$ $C_{l}\left(\mathbb{R}_{+} \times \mathbb{R}\right)$. Therefore, we have

$$
\begin{aligned}
\left\|u_{t}^{n}(\phi)-u_{t}(\phi)\right\|_{\infty} \leq & \left\|R_{n t}^{n}\left(n\left(1-\mathrm{e}^{-\frac{\phi}{n}}\right)\right)-U_{t}(\phi)\right\|_{\infty}+t \| \epsilon_{n}\left(u_{s}^{n}(\phi(0, \cdot)) \|_{\infty}\right. \\
& +2 \lambda c\|\phi\|_{\infty} \int_{0}^{t} \mathrm{~d} s\left\|u_{s}^{n}(\phi)-u_{s}(\phi)\right\|_{\infty} \\
& +\lambda c \int_{0}^{t} \mathrm{~d} s\left\|\left(U_{t-s}-R_{n(t-s)}^{n}\right)\left(u_{s} \phi\right)^{2}\right\|_{\infty} .
\end{aligned}
$$

For $\phi \in C_{l}\left(\mathbb{R}_{+} \times \mathbb{R}\right)$, note that $U_{t}$ is a strongly continuous semigroup which implies that $u_{s}(\phi)$ is a uniformly continuous function. So using Proposition 5.1 the first term and the last term go to zero. By our assumption on $F, \| \epsilon_{n}\left(u_{s}^{n}(\phi(0, \cdot)) \|_{\infty}\right.$ will go to zero as $n \rightarrow \infty$. Now using the standard Gronwall argument we have the result.

\section{PROPOSITION 5.4}

Let $\epsilon>0$. The processes $\mathcal{Y}^{n}$. are tight in $D\left([\epsilon, \infty), M\left(\mathbb{R}_{+} \times \mathbb{R}\right)\right)$.

Proof. By Theorems 3.7.1 and 3.6.5 (Aldous criterion) in [7], it is enough to show

$$
\left\langle\mathcal{Y}_{\tau_{n}+\delta_{n}}^{n}, \phi\right\rangle-\left\langle\mathcal{Y}_{\tau_{n}}^{n}, \phi\right\rangle \stackrel{d}{\longrightarrow} 0,
$$

where $\phi \in C_{l}^{+}\left(\mathbb{R}_{+} \times \mathbb{R}\right), \delta_{n}$ is a sequence of positive numbers that converge to 0 and $\tau_{n}$ is any stopping time of the process $\mathcal{Y}^{n}$ with respect to the canonical filtration, satisfying $0<\epsilon \leq \tau_{n} \leq T$ for some $T<\infty$.

First we note that as $\left\langle\mathcal{Y}_{t}^{n}, 1\right\rangle$ is a martingale, for $\gamma>0$ by Chebyschev's inequality and Doob's maximal inequality we have

$$
P\left(\left\langle\mathcal{Y}_{\tau_{n}}^{n}, \phi\right\rangle>\gamma\right) \leq \frac{1}{\gamma} c_{1}\|\phi\|_{\infty} E\left(\sup _{\epsilon \leq t \leq T}\left\langle\mathcal{Y}_{t}^{n}, 1\right\rangle\right) \leq \frac{1}{\gamma} c_{2}\|\phi\|_{\infty} .
$$

By the strong Markov property applied to the process $\mathcal{Y}^{n}$ we obtain that for $\alpha, \beta \geq 0$, we have

$$
\begin{aligned}
L_{n}\left(\delta_{n} ; \alpha, \beta\right) & :=E\left(\exp \left(-\alpha\left\langle\mathcal{Y}_{\tau_{n}+\delta_{n}}^{n}, \phi\right\rangle-\beta\left\langle\mathcal{Y}_{\tau_{n}}^{n}, \phi\right\rangle\right)\right) \\
& =E\left(\exp \left(-\left\langle\mathcal{Y}_{\tau_{n}}^{n}, u_{\delta_{n}}^{n}(\alpha \phi)+\beta \phi\right\rangle\right)\right) \\
& =E\left(\exp \left(-\left\langle\mathcal{Y}_{\tau_{n}-\epsilon}^{n}, u_{\epsilon}^{n}\left(u_{\delta_{n}}^{n}(\alpha \phi)+\beta \phi\right)\right\rangle\right)\right) .
\end{aligned}
$$

Therefore

$$
\begin{aligned}
& \left|L_{n}(0 ; \alpha, \beta)-L_{n}\left(\delta_{n} ; \alpha, \beta\right)\right| \\
& \quad \leq\left\|u_{\epsilon}^{n}\left(u_{\delta_{n}}^{n}(\alpha \phi)+\beta \phi\right)-u_{\epsilon}^{n}((\alpha+\beta) \phi)\right\|_{\infty} E\left(\sup _{t \leq T}\left\langle\mathcal{Y}_{t}^{n}, 1\right\rangle\right) \\
& \quad \leq c_{1}\left\|u_{\epsilon}^{n}\left(u_{\delta_{n}}^{n}(\alpha \phi)+\beta \phi\right)-u_{\epsilon}^{n}((\alpha+\beta) \phi)\right\|_{\infty},
\end{aligned}
$$


where the last inequality is by Doob's maximal inequality. Now,

$$
\begin{aligned}
& \left\|u_{\epsilon}^{n}\left(u_{\delta_{n}}^{n}(\alpha \phi)+\beta \phi\right)-u_{\epsilon}^{n}((\alpha+\beta) \phi)\right\|_{\infty} \\
& \leq\left\|R_{n \epsilon}^{n}\left(u_{\delta_{n}}^{n}(\alpha \phi)-\alpha \phi\right)\right\|_{\infty} \\
& \quad+c_{2}\|\phi\|_{\infty} \int_{0}^{\epsilon} \mathrm{d} a\left\|u_{a}^{n}\left(u_{\delta_{n}}^{n}(\alpha \phi)+\beta \phi\right)-u_{a}^{n}((\alpha+\beta) \phi)\right\|_{\infty}+d_{n}(\phi),
\end{aligned}
$$

where $d_{n}(\phi)=\lambda \int_{0}^{\epsilon} \mathrm{d} a\left\|\epsilon_{n}\left(u_{a}^{n}\left(u_{\delta_{n}}^{n}(\alpha \phi)+\beta \phi\right)\right)+\epsilon_{n}\left(u_{a}^{n}((\alpha+\beta) \phi)\right)\right\|_{\infty}$. Observe that

$$
\begin{aligned}
&\left\|R_{n \epsilon}^{n}\left(u_{\delta_{n}}^{n}(\alpha \phi)-\alpha \phi\right)\right\|_{\infty} \\
& \leq\left\|R_{n \epsilon}^{n}\left(u_{\delta_{n}}^{n}(\alpha \phi)-R_{n \delta_{n}}^{n}(\alpha \phi)\right)\right\|_{\infty}+\left\|R_{n \epsilon}^{n}\left(R_{n \delta_{n}}^{n}(\alpha \phi)-\alpha \phi\right)\right\|_{\infty} \\
& \leq\left\|u_{\delta_{n}}^{n}(\alpha \phi)-R_{n \delta_{n}}^{n}(\alpha \phi)\right\|_{\infty}+\left\|R_{n\left(\epsilon+\delta_{n}\right)}^{n}(\alpha \phi)-R_{n \epsilon}^{n}(\alpha \phi)\right\|_{\infty} \\
& \leq\left\|R_{n \delta_{n}}^{n}\left(n\left(1-\mathrm{e}^{\frac{\alpha \phi}{n}}\right)-\alpha \phi\right)\right\|_{\infty} \\
&+\int_{0}^{\delta_{n}} \mathrm{~d} a\left\|R_{n\left(\delta_{n}-a\right)}^{n}\left(n^{2} \Psi_{n}\left(\frac{u_{a}^{n}(\alpha \phi)}{n}\right)\right)\right\|_{\infty} \\
&+\left\|R_{n\left(\epsilon+\delta_{n}\right)}^{n}(\alpha \phi)-R_{n \epsilon}^{n}(\alpha \phi)\right\|_{\infty} \\
& \leq\left\|n\left(1-\mathrm{e}^{\frac{\alpha \phi}{n}}\right)-\alpha \phi\right\|_{\infty}+\delta_{n} c_{2}\left(\|\phi\|_{\infty}^{2}+1\right) \\
&+\left\|R_{n\left(\epsilon+\delta_{n}\right)}^{n}(\alpha \phi)-R_{n \epsilon}^{n}(\alpha \phi)\right\|_{\infty} \\
& \equiv e_{n}(\phi) .
\end{aligned}
$$

Consequently,

$$
\begin{aligned}
& \left\|u_{\epsilon}^{n}\left(u_{\delta_{n}}^{n}(\alpha \phi)+\beta \phi\right)-u_{\epsilon}^{n}((\alpha+\beta) \phi)\right\|_{\infty} \\
& \quad \leq e_{n}(\phi)+d_{n}(\phi)+c_{2}\|\phi\|_{\infty} \int_{0}^{\epsilon} \mathrm{d} a\left\|u_{a}^{n}\left(u_{\delta_{n}}^{n}(\alpha \phi)+\beta \phi\right)-u_{a}^{n}((\alpha+\beta) \phi)\right\|_{\infty} .
\end{aligned}
$$

By Proposition 5.1, $e_{n}(\phi) \rightarrow 0$ and $d_{n}(\phi) \rightarrow 0$ by our assumption $F_{n}$. Hence by a standard Gronwall argument we have that

$$
\left|L_{n}(0 ; s, r)-L_{n}\left(\delta_{n} ; s, r\right)\right| \rightarrow 0 .
$$

By (5.15), $\left\{\left\langle\mathcal{Y}_{\tau_{n}}^{n}, \phi\right\rangle ; n=1,2, \ldots\right\}$ is tight in $\mathbb{R}_{+}$. Take an arbitrary subsequence. Then there is a further subsequence of it indexed by $\left\{n_{k} ; k=1,2, \ldots\right\}$ such that $\left\langle\mathcal{Y}_{\tau_{n_{k}}}^{n_{k}}, \phi\right\rangle$ converges in distribution to some random limit $b$. Thus we get

$$
\left(\mathcal{Y}_{\tau_{n_{k}}}^{n_{k}}(\phi), \mathcal{Y}_{\tau_{n_{k}}}^{n_{k}}(\phi)\right) \stackrel{d}{\longrightarrow}(b, b) \quad \text { as } k \rightarrow \infty .
$$

But (5.16) implies that

$$
\left(\mathcal{Y}_{\tau_{n_{k}}}^{n_{k}}(\phi), \mathcal{Y}_{\tau_{n_{k}}+\delta_{n_{k}}}^{n_{k}}(\phi)\right) \stackrel{d}{\longrightarrow}(b, b) \quad \text { as } k \rightarrow \infty .
$$

This implies that $\left\langle\mathcal{Y}_{\tau_{n_{k}}+\delta_{n_{k}}}^{n_{k}}, \phi\right\rangle-\left\langle\mathcal{Y}_{\tau_{n_{k}}}^{n_{k}}, \phi\right\rangle \stackrel{d}{\longrightarrow} 0$ as $k \rightarrow \infty$. So (5.14) holds and the proof is complete. 
Proof of Theorem 1.3. Proposition 5.3 shows that the log-Laplace functionals of the process $\mathcal{Y}_{t}^{n}$ converge to $\mathcal{Y}_{t}$ for every $t \geq \epsilon$. Proposition 5.4 implies tightness for the processes. As the solution to (1.11) is unique, we are done.

\section{Acknowledgements}

Siva Athreya's research was supported in part by CSIR Grant-in-Aid Scheme and Homi Bhabha Fellowship. Srikanth Iyer's research was supported in part by UGC-SAP IV.

\section{References}

[1] Aldous D, Stopping times and tightness, Ann. Probab. 6 (1978) 335-340

[2] Athreya K B and Kang M, Some limit theorems for positive recurrent Markov chains, I and II, Adv. Appl. Probab. 30 (1998) 693-710, 711-722

[3] Athreya K B and Kaplan N, Convergence of the age distribution in the one-dimensional supercritical age-dependent branching process, Ann. Probab. 4(1) (1976) 38-50

[4] Athreya K B and Lahiri S, Probability Theory, 41, TRIM Series (Hindustan Book Agency) (2006)

[5] Athreya K B and Ney P, Branching Processes (New York: Dover) (2000)

[6] Bose A and Kaj I, Age structured super-processes, Technical Report 161 (1991)

[7] Dawson D A, Measure-valued Markov processes, École d'Éte de Probabilités de SaintFlour XXI-1991, 1-260, Lecture Notes in Math. 1541 (Berlin: Springer) (1993)

[8] Dawson D A, Gorostiza L G and Li Z, Nonlocal branching super-processes and some related models, Acta Appl. Math. 74(1) (2002) 93-112

[9] Durrett R, The genealogy of critical branching processes, Stoc. Proc. App. 8 (1978)

[10] Dynkin E B, An introduction to branching measure-valued processes, CRM Monograph Series 6 (American Mathematical Society, Providence, RI) (1994)

[11] Etheridge A, An Introduction to Super-Processes, University Lecture Series 20 (American Mathematical Society, Providence, RI) (2000).

[12] Feller W, An introduction to probability theory and its applications, Vol. II (New YorkLondon-Sydney: John Wiley \& Sons, Inc.) (1966) xviii+636 pp.

[13] Ikeda N, Nagasawa M and Watanabe S, Branching Markov processes, I, J. Math. Kyoto Univ. 8 (1968) 365-410

[14] Kallenberg O, Foundation of Modern Probability Theory (Springer) (2002)

[15] Karlin S and McGregor J, Direct product branching processes and related induced Markov chains I, Calculations of rates of approach to homozygosity, Proc. Internat. Res. Sem. Stat. Lab., Univ. of Calif. Berkeley (California: Springer) (1963) pp. 111-145

[16] Perkins E A, Dawson-Watanabe superprocesses and measure-valued diffusions, Lectures on probability theory and statistics (Saint-Flour) (1999) pp. 125-324, Lecture Notes in Math. 1781 (Berlin: Springer) (2002)

[17] Seneta E and Vere-Jones D, On quasi-stationary distributions in discrete-time Markov chains with a denumerable infinity of states, J. Appl. Probab. 3 (1966) 403-434 